\title{
Compressive Temporal Summation in Human Visual Cortex
}

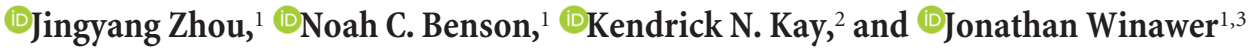 \\ ${ }^{1}$ Department of Psychology, New York University, New York, New York 10003, ${ }^{2}$ Department of Radiology, University of Minnesota-Twin Cities, \\ Minneapolis, Minnesota 55414, and ${ }^{3}$ Center for Neural Science, New York University, New York, New York 10003
}

Combining sensory inputs over space and time is fundamental to vision. Population receptive field models have been successful in characterizing spatial encoding throughout the human visual pathways. A parallel question, how visual areas in the human brain process information distributed over time, has received less attention. One challenge is that the most widely used neuroimaging method, fMRI, has coarse temporal resolution compared with the time-scale of neural dynamics. Here, via carefully controlled temporally modulated stimuli, we show that information about temporal processing can be readily derived from fMRI signal amplitudes in male and female subjects. We find that all visual areas exhibit subadditive summation, whereby responses to longer stimuli are less than the linear prediction from briefer stimuli. We also find fMRI evidence that the neural response to two stimuli is reduced for brief interstimulus intervals (indicating adaptation). These effects are more pronounced in visual areas anterior to V1-V3. Finally, we develop a general model that shows how these effects can be captured with two simple operations: temporal summation followed by a compressive nonlinearity. This model operates for arbitrary temporal stimulation patterns and provides a simple and interpretable set of computations that can be used to characterize neural response properties across the visual hierarchy. Importantly, compressive temporal summation directly parallels earlier findings of compressive spatial summation in visual cortex describing responses to stimuli distributed across space. This indicates that, for space and time, cortex uses a similar processing strategy to achieve higher-level and increasingly invariant representations of the visual world.

Key words: adaptation; fMRI; population receptive fields; temporal summation; visual cortex; visual hierarchy

Significance Statement

Combining sensory inputs over time is fundamental to seeing. Two important temporal phenomena are summation, the accumulation of sensory inputs over time, and adaptation, a response reduction for repeated or sustained stimuli. We investigated these phenomena in the human visual system using fMRI. We built predictive models that operate on arbitrary temporal patterns of stimulation using two simple computations: temporal summation followed by a compressive nonlinearity. Our new temporal compressive summation model captures (1) subadditive temporal summation, and (2) adaptation. We show that the model accounts for systematic differences in these phenomena across visual areas. Finally, we show that for space and time, the visual system uses a similar strategy to achieve increasingly invariant representations of the visual world.

\section{Introduction}

A fundamental task of the visual system is to combine sensory information distributed across space and time. How neural responses sum inputs across space has been well characterized, with

\footnotetext{
Received June 20, 2017; revised Oct. 23, 2017; accepted Nov. 17, 2017.

Author contributions: J.Z., N.C.B., K.N.K., and J.W. designed research; J.Z. and J.W. performed research; J.Z., N.C.B., K.N.K., and J.W. analyzed data; J.Z. and J.W. wrote the paper.

This work was supported by National Institutes of Health Grants R00-EY022116 and R01-MH111417 to J.W. We thank David Heeger, Brian Wandell, and Mike Landy for comments on an earlier draft of this manuscript; and Bosco Tjan, David Heeger, X.J. Wang, Denis Pelli, and Rachel Denison for helpful discussions and feedback as we developed our models and analyses.

The authors declare no competing financial interests.

Correspondence should be addressed to Jingyang Zhou, Department of Psychology, New York University, 6 Washington Place, New York, NY 10003. E-mail: jingyang.zhou@nyu.edu.

DOI:10.1523/JNEUROSCI.1724-17.2017

Copyright $\odot 2018$ the authors $\quad 0270-6474 / 18 / 380691-19 \$ 15.00 / 0$
}

several robust phenomena. First, spatial summation in visual cortex is subadditive: the response to two stimuli presented in different locations at the same time is less than the sum of the responses to the stimuli presented separately. This phenomenon is observed in all cortical areas studied and has been measured with both fMRI (Kastner et al., 2001; Kay et al., 2013a) and electrophysiology (Rolls and Tovee, 1995; Britten and Heuer, 1999; Heuer and Britten, 2002; Winawer et al., 2013); such nonlinearities may reflect an adaptation to achieve efficient encoding of natural images (Schwartz and Simoncelli, 2001). In addition, in higher visual areas, receptive field size increases (Maunsell and Newsome, 1987) and subadditive summation becomes more pronounced (Kay et al., 2013a, b). As the subadditivity becomes more pronounced in later areas and receptive fields get larger, a stimulus that occupies only a small fraction of a neural receptive field can produce a large response. As a result, responses in higher 


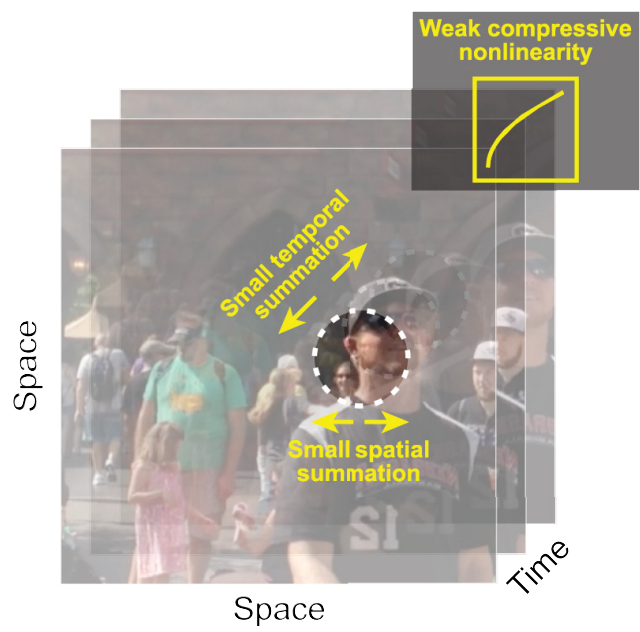

Early visual cortex (V1)

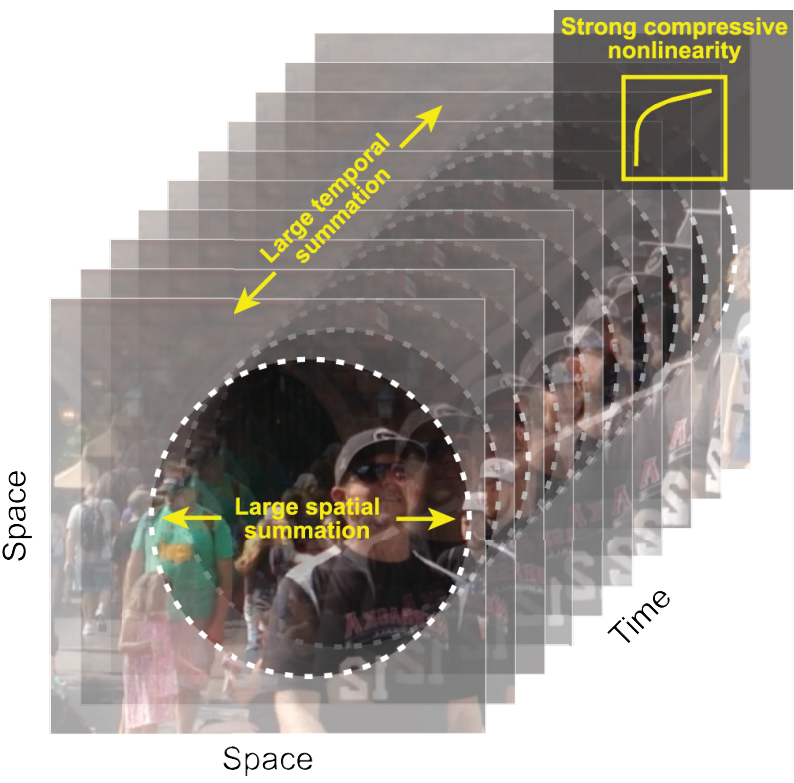

Late visual cortex (TO)

Figure 1. Parallels between spatial and temporal processing. It is well established that spatial receptive fields are small in V1 (left) and grow larger in later visual areas, such as the T0 maps (right). It was recently shown that there is also a gradient of an increasingly pronounced compressive summation over space from early to later areas (Kay et al., 2013a). Here, we hypothesize that temporal summation, as well as the temporal receptive field size, follows a similar pattern, with increasingly long temporal windows and more compressive summation over time in the more anterior visual areas. We propose that the combination of larger spatiotemporal windows and more compressive nonlinearities is part of a coding strategy whereby higher visual areas achieve increasing invariance to changes in stimulus size, position, and duration.

visual areas become increasingly insensitive to changes in the size and position of stimuli (Tovee et al., 1994; Grill-Spector et al., 2001; Kay et al., 2013a). The tendency toward increasing tolerance for size and position in higher areas trades off with the increasing specificity of tuning to higher-level stimulus information (Rust and Dicarlo, 2010, 2012).

Here, we hypothesize that the same organizational principles for the visual cortex apply in the temporal domain (Fig. 1). Just as natural images tend to vary slowly over space, image sequences typically vary slowly over time (Dong and Atick, 1995; Weiss et al., 2002). As a result, an efficient code would prioritize abrupt changes in time over sustained or repeated stimuli (Snow et al., 2016); this would result in subadditive temporal summation for sustained or repeated stimuli (also referred to as adaptation or repetition suppression). Evidence for such temporal nonlinearities are abundant in single-cell recordings of primary visual cortex (e.g., Tolhurst et al., 1980) but have not been systemically characterized across visual areas or with a forward model. At longer time scales, the fMRI BOLD signal sums contrast patterns close to, but slightly less than, linearly (Boynton et al., 1996, 2012). We hypothesize that (1) at the time scale of neuronal dynamics in sensory cortex (tens to hundreds of milliseconds), temporal summation will be substantially subadditive; and (2) more anterior visual areas will show greater subadditivity. This greater subadditivity in later areas will make these responses less sensitive to the precise duration and timing of a stimulus, paralleling size and position tolerance in the spatial domain. This prediction is consistent with the logic that later visual areas trade off position and duration specificity for increased tuning for high-level stimulus properties.

In this paper, we used fMRI to study temporal summation and adaptation. We characterized responses to brief stimuli (tens to hundreds of milliseconds) in many visual areas, measured with
fMRI, which has the advantage of being noninvasive and recording from many visual areas in parallel. To quantify and understand how temporal information is encoded across visual cortex, we implemented a temporal population receptive field ( $\mathrm{pRF}$ ) model, which predicts the fMRI response amplitude to arbitrary stimulus time courses.

\section{Materials and Methods fMRI procedure \\ Participants}

Data from 6 experienced fMRI participants (2 males and 4 females, age range 21-48 years, mean age 31 years) were collected at the Center for Brain Imaging at New York University. All participants had normal or corrected-to-normal visual acuity. The experimental protocol was approved by the University Committee on Activities Involving Human Subjects, and informed written consent was obtained from all participants before the study. For each participant, we conducted a $1 \mathrm{~h}$ session for visual field map identification and high-resolution anatomical volumes, and either one or two $1.5 \mathrm{~h}$ sessions to study temporal summation. Two of the 6 participants ( 1 male, 1 female) were included in both the main temporal summation experiment and the self-replication experiment (hence, two $1.5 \mathrm{~h}$ sessions). The other 4 participants ( 1 male and 3 females) were included in only the main temporal summation experiment or the self-replication experiment.

\section{Visual stimuli}

Stimuli. For the main experiment, stimuli were large-field ( $24^{\circ}$ diameter) bandpass noise patterns (centered at 3 cycles per degree), independently generated for each trial. The pattern was chosen because it was previously shown to be effective in eliciting responses in most visual areas (Kay et al., 2013b; for details on stimulus construction, see Kay et al., 2013b). A second experiment replicated all aspects of the main experiments, except that the stimulus patterns differed. For this experiment, the patterns were either pink noise ( $1 / \mathrm{f}$ amplitude spectrum, random phase) or a frontview face image embedded in the pink noise background. The face stim- 
uli were the front-facing subset of the faces used by Kay et al. (2015). For both experiments, stimuli were windowed with a circular aperture $\left(24^{\circ}\right.$ diameter, $768 \times 768$ pixels) with a raised cosine boundary $\left(2 \cdot 4^{\circ}\right)$. All stimuli were grayscale. Stimulus generation, presentation, and response recording were coded using Psychophysics Toolbox (Brainard, 1997; Pelli, 1997) and vistadisp (https://github.com/vistalab/vistadisp). We used a MacBook Air computer to control stimulus presentation and record responses from the participants (button presses) during the experiment.

Display. Stimuli were displayed via an LCD projector (Eiki LC_XG250; resolution: $1024 \times 768$ pixels; refresh rate: $60 \mathrm{~Hz}$ ) onto a back-projection screen in the bore of the magnet. Participants, at a viewing distance of $\sim 58 \mathrm{~cm}$, viewed the screen (FOV, horizontal: $\sim 32^{\circ}$, vertical: $\sim 24^{\circ}$ ) through an angled mirror. The images were confined to a circular region with a radius of $12^{\circ}$. The display was calibrated and gamma corrected using a linearized lookup table.

Fixation task. To stabilize attention level across scans and across participants during the main experiment, all participants were instructed to do a one-back digit task at the center of fixation throughout the experiment, as in previous publications (Kay et al., 2013a, b). The digit $\left(0.24^{\circ} \times\right.$ $\left.0.24^{\circ}\right)$ was presented at the center of a neutral gray disk $\left(0.47^{\circ}\right.$ diameter $)$. Within a scan, each digit (randomly selected from 0 to 9 ) was on for $0.5 \mathrm{~s}$, off for $0.167 \mathrm{~s}$ before the next digit appeared at the same location. Participants were asked to press a button when a digit repeated. Digit repetition occurred at $\sim 2 \%-3 \%$, with no more than two identical digits being presented successively. To reduce visual adaptation, all digits alternated between black and white, and on average participants pressed a button every $30 \mathrm{~s}$. During the retinotopy task, the fixation alternated pseudorandomly between red and green (switches on average every $3 \mathrm{~s}$ ), and the participant pressed a button to indicate color changes.

\section{Experimental design}

We used a randomized event-related experimental design (see Fig. $2 A, B$ ) to prevent participants from anticipating the stimulus conditions. An event is a stimulus presented according to 1 of 13 distinct time courses $(<800 \mathrm{~ms}$ in total), either a single pulse with variable duration or a double pulse with fixed duration and variable interstimulus interval (ISI). Durations and ISIs were powers of 2 times the monitor dwell time $(1 / 60 \mathrm{~s})$. Each pulse in the double-pulse stimuli lasted $134 \mathrm{~ms}$. The $0 \mathrm{~ms}$ stimulus was a blank (zero-contrast, mean luminance, and hence identical to the preceding and subsequent blank screen between stimulus events). For the main experiment, each participant completed 7 scans; and within a scan, each temporal event repeated 4 times. A temporal event started with the onset of a pattern image, and the intertrial interval (stimulus plus subsequent blank) was always $4.5 \mathrm{~s}$. For stimuli with two pulses, the two noise patterns were identical. The design was identical for the self-replication experiment, except that each time course repeated 3 times per scan instead of 4 , and each participant completed 6 scans.

\section{MRI data acquisition}

All fMRI data were acquired at New York University Center for Brain Imaging using a Siemens Allegra 3T head-only scanner with a Nova Medical phased array, 8-channel receive surface coil (NMSC072). For each participant, we collected functional images ( $1500 \mathrm{~ms}$ TR, $30 \mathrm{~ms}$ TE, and $72^{\circ}$ flip angle). Voxels were $2.5 \mathrm{~mm}^{3}$ isotopic, with 24 slices. The slice prescription covered most of the occipital lobe, and the posterior part of both the temporal and parietal lobes. Images were corrected for B0 field inhomogeneity using Center for Brain Imaging algorithms during offline image reconstruction.

In a separate session, we acquired 2 or $3 \mathrm{~T} 1$ weighted whole-brain anatomical scans (MPRAGE sequence; $1 \mathrm{~mm}^{3}$ ). Additionally, a T1 weighted "inplane" image was collected with the same slice prescription as the functional scans to aid alignment of the functional images to the highresolution $\mathrm{T} 1$ weighted anatomical images. This scan had an inplane resolution of $1.25 \times 1.25 \mathrm{~mm}$ and a slice thickness of $2.5 \mathrm{~mm}$.

\section{Data preprocessing and analysis}

Data preprocessing. We coregistered and segmented the T1 weighted whole-brain anatomical images into gray and white matter voxels using FreeSurfer's autosegmentation algorithm (http://surfer.nmr.mgh.harvard. edu/). Using custom software vistasoft (https://github.com/vistalab/ vistasoft), the functional data were slice-time corrected by resampling the time series in each slice to the center of each $1.5 \mathrm{~s}$ volume. Data were then motion-corrected by coregistering all volumes of all scans to the first volume of the first scan. The first 8 volumes $(12 \mathrm{~s})$ of each scan were discarded for analysis to allow longitudinal magnetization and stabilized hemodynamic response.

GLM analysis. For analysis of the temporal summation functional data, we used a variant of the GLM procedure, GLM denoise (Kay et al., 2013c), a technique that improves signal-to-noise ratios by entering noise regressors into the GLM analysis. Noise regressors were selected by performing principle component analysis on voxels whose activities were unrelated to the task. The optimal number of noise regressors was selected based on cross-validated $R^{2}$ improvement (coefficient of determination). The input to GLM denoise was the preprocessed EPI data and a design matrix for each scan (13 distinct temporal profiles $\times$ number of volumes per scan), and the output was $\beta$ weights for each temporal profile for each voxel, bootstrapped 100 times across scans (see Fig. 2B). For analysis, we normalized all $13 \beta$ weights per voxel by the vector length and selected a subset of voxels (see Voxel selection). We then averaged the $\beta$ weights for a given temporal condition from the first bootstrap across voxels within each region of interest (ROI) and across all participants to get a mean; this gives one estimate of the mean response per ROI for a given condition. This was repeated for each condition and then repeated for each of the 100 bootstraps, yielding a matrix of $100 \times 13$ for each ROI (bootstraps by temporal condition). GLM denoise was not applied to the visual field map measurements because these experiments did not have an event-related design, and hence are not amenable to a GLM analysis.

ROI identification. We fitted a linear population (pRF) model (Dumoulin and Wandell, 2008) to each subject's retinotopy data (average of 2 scans). We made an initial guess of ROI locations by first projecting the maximum likelihood probabilistic atlas from Wang et al. (2015) onto the cortical surface. Then we visualized eccentricity and polar angle maps derived from the pRF model fits and modified ROI boundaries based on visual inspection. For each participant, we defined nine bilateral ROIs (V1, V2, V3, hV4, VO-1/2, LO-1/2, TO-1/2, IPS-0/1; see Fig. 2C). For the second experiment (self-replication), in addition to the nine ROIs from the main experiment, we also identified a bilateral face-selective ROI. This ROI included face-selective voxels in the inferior occipital gyrus (IOG faces, or occipital face area) and in the posterior fusiform (pFus, or fusiform face area-1) (Gauthier et al., 2000; Weiner and Grill-Spector, 2010). We identified these areas by taking the difference between the mean fMRI response to all face images and the mean response to all noise images, and then thresholding the difference for voxels at the two anatomical locations (inferior occipital gyrus and posterior fusiform), as described previously (Weiner and Grill-Spector, 2010; Kay et al., 2015).

Voxel selection. All analyses were restricted to voxels that satisfy the following three criteria. First voxels must be located within $2^{\circ}-10^{\circ}$ (eccentricity) based on the pRF model. Second, voxels must have a positive $\beta$ weight for the average across all nonblank temporal conditions (and averaged across bootstraps). The bootstraps, computed by GLM denoise, were derived by sampling with replacement from the repeated scans. Third, voxels must have $>2 \%$ GLM $R^{2}$. Voxels that satisfy all criteria were averaged within a participant to yield $13 \beta$ weights per ROI per participant per 100 bootstraps. The data were then averaged across participants. Averaging within a participant before averaging across participants ensures that the contribution for each participant has the same weight, regardless of the numbers of voxels per participant.

\section{Hemodynamic response function (HRF) for individual ROIs}

In the section, The CTS model is more accurate than a two temporal channels model in later visual areas, we estimate an HRF for each ROI to test whether the use of ROI-specific HRFs, rather than a single HRF for each subject, altered the pattern of results. We estimated the HRFs using two experiments: the retinotopic mapping experiment and the temporal summation experiment. In both cases, we assumed that the HRF was parameterized by the difference between two gamma functions with five free parameters (Friston et al., 1998; Worsley et al., 2002). For the retinotopy HRF, we used the vistasoft pRF code, which uses an iterative 
approach alternating between fitting the pRF parameters and the HRF parameters: first, the HRF is assumed to have default parameters for all voxels and the pRF parameters are fit; then the pRF parameters are fixed and the HRF is found, which maximizes the mean variance explained across voxels in an ROI; finally, the HRF parameters are fixed and the pRF parameters are refit. This procedure was done separately for each ROI.

To estimate HRFs from the temporal experiment, we first selected voxels for each ROI as described in Voxel selection. We averaged the output time series from GLMdenoise (0-mean, polynomial detrended, and noise PCs regressed-out) from the selected voxels within each ROI to estimate a set of $\beta$ weights. As with the retinotopy experiment, we estimated the HRF and a set of $\beta$ weights for each ROI using an iterative procedure. Each HRF was parameterized using the difference between two gamma functions with five free parameters (same as for retinotopy), and was seeded using the same vistasoft default parameters (see rmHrfTwogamms.m). The iterative fitting procedure terminates when the two types of fits converge.

\section{Temporal pRF models}

We used two variants of a temporal pRF model, one linear and one nonlinear, to predict neuronal summation measured using fMRI. All model forms take the time course of a spatial contrast pattern as input $\left(T_{\text {input }}\right)$, and produce a predicted neuronal response time course as output. To predict the fMRI data (BOLD), we summed the predicted time course within a trial $(<1 \mathrm{~s})$ to yield one number per temporal condition. For model fitting, these numbers were compared with the fMRI $\beta$ weights, derived from the GLM denoise analysis.

\section{Models}

Linear model. The linear model prediction is computed by convolving a neuronal impulse response function (IRF) with the stimulus time course $\left(T_{\text {input }}\right)$, and scaling by a gain factor $(g)$ as follows:

$$
R_{\text {linear }}=g\left[\operatorname{IRF} * T_{\text {input }}\right]
$$

The time course is then summed for the fMRI predictions (plus an error term, $e$ ) as follows:

$$
B O L D_{\text {linear }}=\sum g\left[I R F * T_{\text {input }}\right]+e
$$

For the IRF, we assumed a gamma function, parameterized by $\tau_{1}$, of the form,

$$
I R F=t * \exp (-t / \tau)
$$

Because the IRF was assumed to have unit area, the specific shape of the IRF has no effect on the predictions of the linear model, and the prediction reduces to the following:

$$
B O L D_{\text {linear }}=g\left[\sum T_{\text {input }}\right]+e
$$

and the only value solved for is the gain factor, $g$.

Compressive temporal summation model (CTS). The CTS model is computed with a linear convolution, followed by a divisive normalization. The linear step is identical to the linear model. For the divisive normalization:

$$
\begin{aligned}
B O L D_{C T S} & =g \sum \frac{x^{2}}{\sigma^{2}+x^{2}}+e \\
x & =\operatorname{IRF}(\tau) * T_{\text {input }}
\end{aligned}
$$

we solved for $\tau, \sigma$, and gain factor $g$ for the CTS model. Additionally, we implement two variations of this model. In the first variation, we relaxed the exponent from 2 to $n$, and fitted 4 parameters, $\tau, \sigma, n$, and the gain factor $g$, as follows:

$$
B O L D_{C T S}=g \sum \frac{x^{n}}{\sigma^{n}+x^{n}}+e
$$

In the second variation, we allowed the exponent in the numerator to be different from that in the denominator, and we fitted 5 parameters, $\tau, \sigma$, $n, m$, and $g$, as follows:

$$
B O L D_{C T S}=g \sum \frac{x^{m}}{\sigma^{n}+x^{n}}+e
$$

CTS with power-law implementation. In Alternative implementations of CTS model, we implement the CTS model with a power-law nonlinearity rather than divisive normalization. To compute the model predicted neuronal response, we first computed the linear response by convolving an IRF (gamma function with variable time to peak $\tau$ ) with an input stimulus time course, identical to the normalization implementation. Then, an exponent $\varepsilon$ is applied point-wise to the predicted linear output as follows:

$$
R_{\text {CTS }}=g\left[\operatorname{IRF}(\tau) * T_{\text {input }}\right]^{\varepsilon}
$$

To fit the CTS model with a power law to the fMRI data, we again summed the predicted response time series as follows:

$$
B O L D_{C T S}=g \sum\left[\operatorname{IRF}(\tau) * T_{\text {input }}\right]^{\varepsilon}+e
$$

and solved for $\tau_{1}, \varepsilon$, and $g$.

Because of the nonlinearity, the specific shape of the IRF does matter, in contrast to the linear model. This version of the CTS model is identical to the normalization implementation, except that the shape of the compressive nonlinearity due to the power law is slightly different from the shape obtained using divisive normalization.

Two temporal channel (TTC) model. We implemented a TTC model, previously used for fitting V1 responses to temporal variation in luminance (Horiguchi et al., 2009). The TTC model consists of a linear combination of the outputs from a sustained and a transient temporal channel. The output of the sustained channel is computed by convolving an IRF with the stimulus. The output of the transient channel is computed by convolving the transient IRF with the stimulus time course, then squared point-wise in time. The sustained IRF has a positive mean and the transient IRF has a zero mean, as implemented by Horiguchi et al. (2009) for fMRI data. This IRF form was previously used for modeling psychophysical data (Watson, 1982; McKee and Taylor, 1984). The outputs from both channels are weighted by parameter $a$ and $b$ as follows:

$$
B O L D_{T T C}=\sum\left\{a\left[I R F_{\text {sustained }} * T_{\text {input }}\right]+b\left[I R F_{\text {transient }} * T_{\text {input }}\right]^{2}\right\}
$$

The form of both the sustained and the transient channel IRFs is the same as follows:

$$
I R F_{T T C}=\left(\frac{t}{x}\right)^{8} e^{-t / x}-\frac{1}{y}\left(\frac{t}{z}\right)^{9} e^{-t / z}
$$

with $t$ being time, and $x, y$, and $z$ take values $(3.29,14$, and 3.85), respectively for the sustained IRF, and $(2.75,11,3.18)$ for the transient IRF.

\section{Parameter estimation and model fitting}

All models except the linear model were fit in two steps, a grid fit followed by a search fit, as described below (see also script trf_fitModel.m).

CTS model-normalization implementation. For the grid fit, we computed the model response to the 13 temporal conditions for 100 combinations of $\tau$ and $\sigma$ ( $\tau$ values from $10^{-3}$ to 1 with 10 equal steps, and $\sigma$ from $10^{-3}$ to 0.5 with 10 equal steps). For each ROI, the parameter pair generating the predictions with the highest correlation to the data was then used as a seed for the search fit. This was repeated 100 times per ROI, once for each bootstrap of the data (see trf_gridFit.m and trf_fineFit.m).

We then did a search fit using a bounded nonlinear search algorithm in MATLAB (The MathWorks; fminsearchbnd.m), 100 times per ROI, using the 100 sets of bootstrapped $\beta$ weights, and the 100 seed values as derived above. The search finds the parameters that minimize the squared error between predicted and measured $\beta$ weights. The lower bound used for the search fit is $\left(10^{-3}, 10^{-4}, 0\right)$ for $\tau, \sigma$, and $g$, a scaling factor. The upper bound was $(1,1,1)$. This gave us 100 estimates of each model parameter for each ROI, which we summarized by the median and 50\% CI. 
Additionally, for Alternative implementations of CTS model, we implement the normalization model with additional free parameters. To fit the first variation of the normalization model (free parameters: $\tau, \sigma, n, g$ ), we used the same 10 steps grid fit for $\tau$ and $\sigma$ as in the previous model. For $n$, a 10 step grid with equal steps from 0.1 and 6 was used. In the search fit stage, same bound was used for $\tau, \sigma$, and $g$ as in the previous model, and the bound for $n$ was $(0,10)$. To fit the second variation of the normalization model (free parameters: $\tau, \sigma, n, m, g$ ), the same 10 step grid was used for both $n$ and $m$ in the search stage, and the same bound for both exponents was used in the search stage.

CTS model-exponential implementation. For the grid fit, we computed the model responses to the 13 temporal conditions for 100 combinations of $\tau$ and $\varepsilon$ ( $\tau$ values from $10^{-3}$ to 1 with 10 equal steps; $\varepsilon$ values from $10^{-3}$ to 2 with 10 equal steps). The procedure to select the best fitting parameter pair and the search fit step is the same as above. The lower bound we used for the search fit is $(0.02,0.001,0)$ for $\tau, \varepsilon$, and $g$ (a scaling factor). The upper bound was $(1,2,1)$ (see trf_gridFit.m and trf_fineFit.m).

TTC model. For the grid fit, we generated a $10 \times 10$ grid for the sustained and the transient weight (from $10^{-5}$ to 1 with 10 equal steps). Then we implemented the search fit step as above, with lower bound $(0$, 0 ) and upper bound $(1,1)$ (see trf_gridFit.m and trf_fineFit.m).

Linear model. The linear model does not require a search or seeds. Instead, we fit the 100 bootstrapped datasets per ROI by linear regression, giving us 100 estimates of the gain factor, $g$, per ROI.

\section{Statistical analysis}

We compared model accuracy of the CTS and the linear model. Because the models have different numbers of free parameters, it is important to obtain an unbiased estimate of model accuracy, which we did by leaveone-out cross validation. For each ROI, and for each of the 100 bootstrapped sets of $\beta$ weights, we fit 13 linear models and 13 CTS models by leaving out each of the 13 temporal stimuli. For each bootstrap, we thus obtained 13 left-out predictions, which were concatenated and compared with the $13 \beta$ weights by coefficient of determination, $R^{2}$ as follows:

$$
R^{2}=100 \times\left[1-\frac{\sum(M O D E L-D A T A)^{2}}{\sum D A T A^{2}}\right]
$$

This yielded $100 R^{2}$ values per ROI, and we summarized model accuracy as the median and $50 \% \mathrm{CI}$ derived from these 100 values.

The coefficient of determination, $R^{2}$, is bounded by $(-\infty, 1)$, as the residuals between model and data can be larger than the data. In contrast, $r^{2}$ is bounded by $(0,1)$.

Noise ceilings. The noise ceiling represents the highest accuracy a model can achieve given the signal-to-noise ratio in the data, regardless of the specific model used. We computed noise ceilings stochastically based on the mean and SE of the GLM- $\beta$ weights from bootstrapped estimates, as implemented by Kay et al. (2013a).

Flat model. We computed a model that assumes that the BOLD responses to all stimuli are identical ("flat model") as a further basis of comparison with the CTS and linear models. Like the CTS and the linear model, the accuracy of the flat model was computed by leave-one-out cross validation. The cross-validated predictions from the flat model are not quite identical across conditions, because the mean is affected by the left-out data.

Parameter recovery. To estimate how well model parameters are specified, for each visual area, we simulated the CTS model responses by first generating the predicted fMRI $\beta$ weight for each temporal condition. The parameters used for simulation were the median of each of the parameters from the bootstrapped fits to the data. We then added noise to each $\beta$ weight by randomly sampling from a normal distribution whose SD was matched to the SE in the bootstrapped data, averaged across the temporal conditions. We added noise 1000 times per ROI and then solved the CTS model for the 1000 simulated responses using the same procedure used with the actual data. The parameters recovered from this fitting procedure provide an estimate of how well specified each parameter is given the form of the model (including the parameters) and the noise level in the data.

\section{Public datasets and software code}

To ensure that our computational methods are reproducible, all data and all software are made publicly available via an open science framework site: https://osf.io/v843t/. The software repository includes scripts of the form trf_mkFigure2 to reproduce Figure 2, etc., as in prior publications (e.g., Winawer and Parvizi, 2016).

\section{Results}

\section{Measuring temporal summation in visual cortex}

In each trial of the experiment, participants viewed either one or two pulses of a static spatial contrast pattern. The pattern was an independently generated bandpass noise image (24 diameter), used in prior studies of spatial encoding (Kay et al., 2013a, b). For the two-pulse trials, the two spatial patterns were identical. Each trial used 1 of 13 distinct time courses (Fig. 2A). The durations of the one-pulse stimuli and the ISIs of the two-pulse stimuli were the same: $0,1,2,4,8,16,32$, or 64 video frames of a $60 \mathrm{~Hz}$ monitor (i.e., 0, 17, 33, 67, 134, 267, $533 \mathrm{~ms}$ ). Each pulse in the 2-pulse stimuli was 8 frames $(134 \mathrm{~ms})$. The $0 \mathrm{~ms}$ one-pulse stimulus was a blank (mean luminance), and the two-pulse stimulus with 0 ISI was identical to the one-pulse stimulus of twice the length (16 frames, $267 \mathrm{~ms}$ ). Four participants were scanned, and data were binned into nine bilateral, eccentricity-restricted $\left(2^{\circ}-10^{\circ}\right)$ visual areas defined from a separate retinotopy scan (Fig. 2C).

The fMRI data were analyzed in two stages. First, we extracted the amplitude ( $\beta$ weight) for each of the 13 temporal conditions using a variation of the GLM denoise (Kay et al., 2013c), a technique that improves the signal-to-noise ratio by including noise regressors in the GLM (Fig. $2 B$ ). Second, we fitted the temporal pRF model to the GLM $\beta$ weights, averaged across voxels within ROIs.

\section{Temporal summation in visual cortex is subadditive}

We tested the linearity of the fMRI BOLD signal in each visual area. To do so, we assume a time-invariant linear system such that the BOLD amplitude (GLM $\beta$ weight) is proportional to the total stimulus duration within the trial. Because of the linearity assumption, the form of the neural IRF does not affect the pattern of the predicted BOLD amplitudes. For example, the linear prediction is that a stimulus of duration $2 t$ produces twice the amplitude as a stimulus of duration $t$, and the same amplitude as a two-pulse stimulus, with total duration $2 t$ (Fig. $3 A$ ). This prediction is not borne out by the data. The response to a stimulus of length $2 t$ is $\sim 75 \%$ of the linear prediction in $\mathrm{V} 1$ and $50 \%$ in area TO (a homolog of macaque areas MT and MST) (Fig. 3B, left). This systematic failure of linearity is found in all visual areas measured, with temporal summation ratios $<0.8$ for all ROIs, and a tendency toward lower ratios in later areas (Fig. $3 C$ ). The BOLD amplitudes to all stimuli are low $(<1 \%)$ because the stimuli are brief, compared with measurements of visual cortex using moving stimuli or a block design with multiple static stimuli, where percentage BOLD changes can be several percent.

A further failure of linearity occurs for trials with two pulses and variable ISI: the response is larger when the ISI is longer, especially in V1. The linear prediction is that the amplitudes are the same, and double the response to the one-pulse stimulus (Fig. $3 B$, right). When the ISI is relatively long $(528 \mathrm{~ms})$, the response in $\mathrm{V} 1$ is close to the linear prediction made from the one-pulse stimulus. In TO, even with a long ISI, the response is still well below the linear prediction. This pattern, whereby the response to a second stimulus is reduced for short ISIs, and larger for longer ISIs, is often called adaptation and recovery (Priebe et al., 2002; Kohn, 2007). For TO, the recovery time is longer than V1, and longer than the longest ISI we tested. 


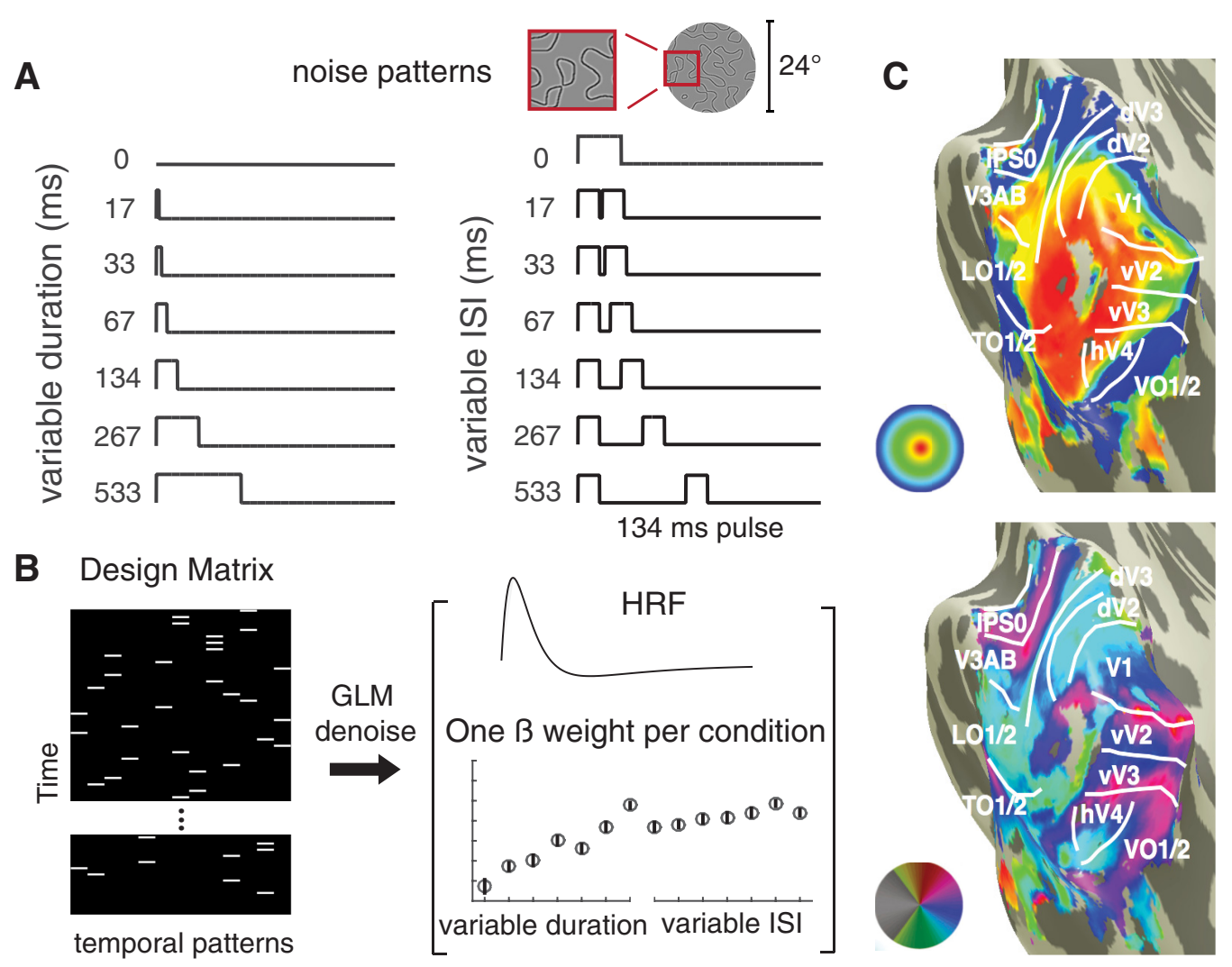

Figure 2. Experimental design and analysis. $A$, Participants were presented with one or two pulses of large field $\left(24^{\circ}\right)$ spatial contrast patterns. One-pulse stimuli were of varying durations, and two-pulse stimuli were of varying $\mid S I$ ( with each pulse lasting $134 \mathrm{~ms}$ ). $\boldsymbol{B}$, The temporal conditions were presented in random order, indicated by the white bars in the 13 -column design matrix (one column per temporal condition). To analyze the data, we extracted a $\beta$ weight for each temporal condition per area using a variant of the GLM denoise. $C$, Nine visual field maps or visual field maps pairs were bilaterally identified for each participant (V1; V2; V3; hV4; V0-1/2; V3A/B; IPS-0/1; L0-1/2; TO-1/2).

The temporal subadditivity is captured by a CTS model We modeled the temporal subadditivity with a CTS model (Fig. $4 A$ ). The CTS model has a linear-nonlinear structure. The linear stage convolves the stimulus time course with a temporal IRF (parameterized by the time constant $\tau$ ). The nonlinear stage passes the linear output through a static nonlinearity, divisive normalization. The normalization is implemented by squaring the linear response at each time point (as in Heeger, 1992), and dividing this by the sum of two terms, a semisaturation constant $(\sigma)$ and the linear response, both of which are squared (Heeger, 1992). Squaring is widely used for modeling neural computations, such as color (Helmholtz, 1886; Koenderink et al., 1972) and motion (Adelson and Bergen, 1985; Simoncelli and Heeger, 1998). The normalization model was developed to describe responses at the level of single neurons. However, we can generalize it to an fMRI voxel by assuming that the neurons within a voxel share a normalization pool, and the voxel sums across neurons within it. In this case, the normalization equation has the same term in the numerator and denominator, as implemented in the CTS model (see also Kay et al., 2013a).

We illustrate the effect of the CTS model with example responses to brief stimuli (17 and $33 \mathrm{~ms}$ ), assuming an IRF with time constant $100 \mathrm{~ms}$ (Fig. 4B). For a linear model, the predicted response to the longer stimulus peaks at almost double the value of the briefer stimulus. For the CTS model with a large $\sigma$, the response more than doubles for the long stimulus compared with the brief stimulus, due to the squaring in the numerator. When $\sigma$ is small, the model is compressive, as the response to the longer stimulus is very similar to the brief stimulus.
To relate the CTS model output to the BOLD signal, we summed the predicted CTS output for a trial, and scaled this by a gain parameter, $g$, to convert to units of percentage BOLD change. We sum the CTS output to give a single value per temporal condition, which can be compared with the $\beta$ weight in each condition, fit from the GLM. If we instead convolve the time-varying CTS model prediction with an HRF, rather than convolving the summed CTS model prediction with the HRF, the predicted BOLD response is nearly identical (Fig. $4 C$ ). We note that while we refer to the model as compressive, technically the normalization model amplifies the output when the instantaneous linear response is low due to the squaring in the numerator, and compresses the response when the amplitude is high. However, for all temporal conditions we tested, the model output is compressive in the sense that the predicted response for any of our single pulse stimuli is less than the linear prediction from a briefer stimulus.

We compared the CTS model with a linear model by measuring cross-validated accuracy. The CTS model is more accurate than the linear model for all areas (Fig. 5). The linear model substantially underpredicts responses to short durations and overpredicts responses to long durations, whereas the CTS model does not. Further, the predictions of the linear model do not depend on ISI, whereas the CTS model correctly predicts that the response amplitude increases with longer ISI. The cross-validated CTS model predicts the left-out fMRI responses with accuracy between $81 \%$ and $98 \%$ across the 9 ROIs. This represents a large improvement compared with the linear model for every area (Fig. $5 B)$. The improvement is especially pronounced in later than early areas (LO/TO/IPS vs V1-V3). 

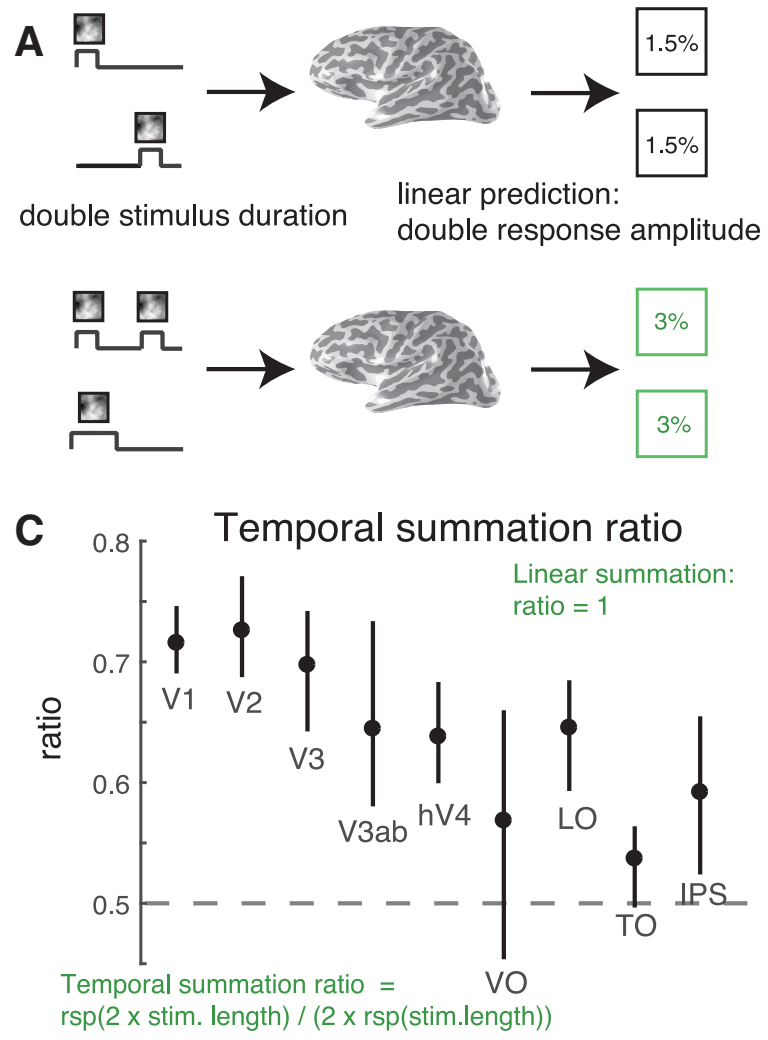
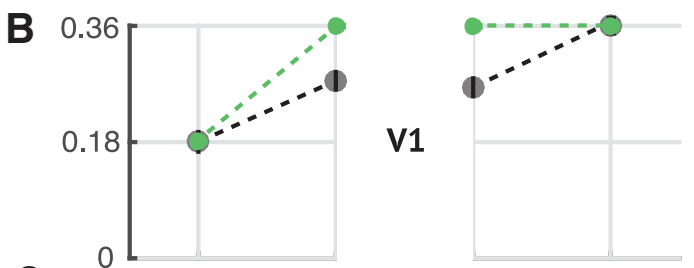
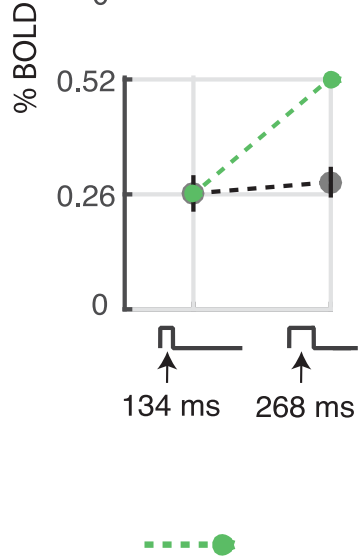

linear prediction based on response to 134 ms stimulus
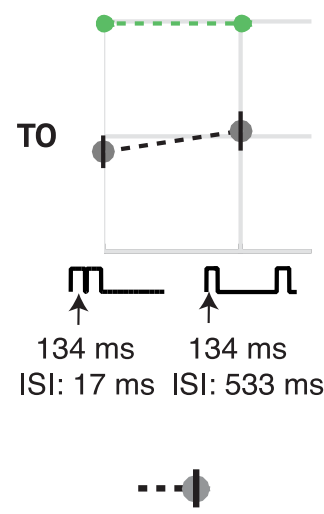

data ( $\beta$-weight measured at each temporal condition)

Figure 3. Sublinear temporal summation in visual cortex. $A$, Linear temporal summation prediction. The sum of the responses to two separate events (top) is equal to the response to the two events in the same trial, with or without a brief gap between them (bottom). $\boldsymbol{B}$, Sublinear temporal summation. Gray circles represent the measured responses to a $134 \mathrm{~ms}$ pulse, a $268 \mathrm{~ms}$ pulse, and two 134 ms pulses, with either a 17 or 134 ms gap between pulses. Plots represent the mean across participants and $50 \% \mathrm{Cl}$ (bootstrapped across repeated scans within each participant). Green circles and dotted lines represent the linear prediction based on the response to the single $134 \mathrm{~ms}$ pulse. For V1, the measured responses are less than the linear prediction, except when there is a long gap. For area $\mathrm{T} 0$, all responses are less than the linear prediction. C, Temporal summation ratio. Temporal summation ratio is the response to a stimulus of length $2 x$ divided by twice the response to a single pulse stimulus of length $x$, averaged across 5 stimulus pairs (17 and $34 \mathrm{~ms}, 34$ and $68 \mathrm{~ms}$, etc). Linear summation occurs when the temporal summation ratio is 1 . The temporal summation ratio is $<1$ in all visual areas, indicating compressive temporal summation. The ratio is higher in early visual areas $(\sim 0.7$ in V1-V3) and lower in later areas $(\sim 0.5$ to 0.65$)$. Error bars indicate the $50 \% \mathrm{Cl}$ (bootstrapped across scans). The ROIs on the $x$-axis are arranged in order of increasing spatial pRF size at 5 degrees eccentricity, as a proxy for order in the visual hierarchy. Figure made from the script trf_mkFigure3.m.

The CTS model is also more accurate than a flat model (Fig. $5 B)$. The flat model predicts the same response amplitude to all stimuli. This indicates that, although the BOLD responses are relatively small (low percentage signal change) and compressive (similar for different duration stimuli), there are nonetheless meaningful differences in the response amplitudes to different temporal conditions. Importantly, the CTS model accurately captures these differences, as it is substantially more accurate than the flat model. One notable exception is area TO, where the BOLD responses are most compressive: here the CTS model is only slightly more accurate than the flat model (and both are much more accurate than the linear model). In contrast, the linear model is more accurate than the flat model only in early visual areas (V1-V3) and less accurate in higher visual areas.

Overall, all three models have a high cross-validated accuracy (from $\sim 70 \%$ to $98 \%$ ). There are several factors to consider in these summary metrics. First, the analysis takes place in two stages: an initial general linear model that yields one coefficient per condition, and then a model fitted to these coefficients. The model accuracy is computed on the 13 coefficients, not the complete BOLD time series, for which the model accuracy would be lower. Second, the model accuracy is computed per ROI, not per voxel, attenuating the noise in individual voxels. Finally, the metric we used to summarize accuracy, $R^{2}$, or coefficient of determination (see formula in Statistical analysis), is influenced by the degree to which the model predictions get the mean right across conditions. This contrasts with, for instance, the squared Pearson correlation $\left(r^{2}\right)$, which subtracts the mean from both the model predictions and the data. We do not subtract the means because getting the mean right is part of the model (gain parameter, $g$ ). For all of these reasons, it is important to compare the $R^{2}$ across the models (linear, CTS, flat). Doing so shows that the CTS model is most accurate in all ROIs.

Finally, we note that, although the cross-validated accuracy of the CTS model is high (close to the noise ceiling in all areas), some data points appear to deviate systematically from the model predictions; for example, the response to the $17 \mathrm{~ms}$ single-pulse stimulus is underpredicted in $\mathrm{TO}$, and the $67 \mathrm{~ms}$ single pulse stimuli are underpredicted in multiple areas. We do not try to interpret these particular data points as they were not robust to replication (see The CTS model fits replicate across experiments).

\section{The CTS model fits capture systematic differences between areas}

The CTS model is parameterized by $\tau, \sigma$, and a gain factor, $g . \tau$ is the time to peak in the temporal IRF and therefore is related to temporal summation window length; $\sigma$ is the semisaturation constant and reflects how much the CTS prediction deviates from the linear prediction. When $\sigma$ is lower, the response is more compressive. The intuition for this is that, when $\sigma$ is small, the numera- 
A Compute linear Rsp. ( $\mathrm{\tau})$

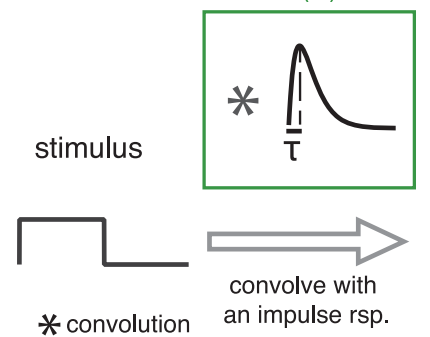

Compute normalized Rsp.

$(\sigma)$

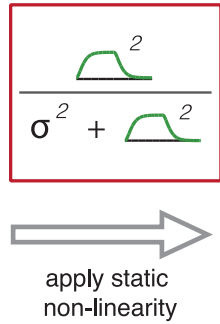

convert to fMRI

(g)

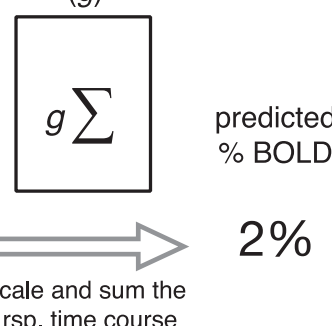

B
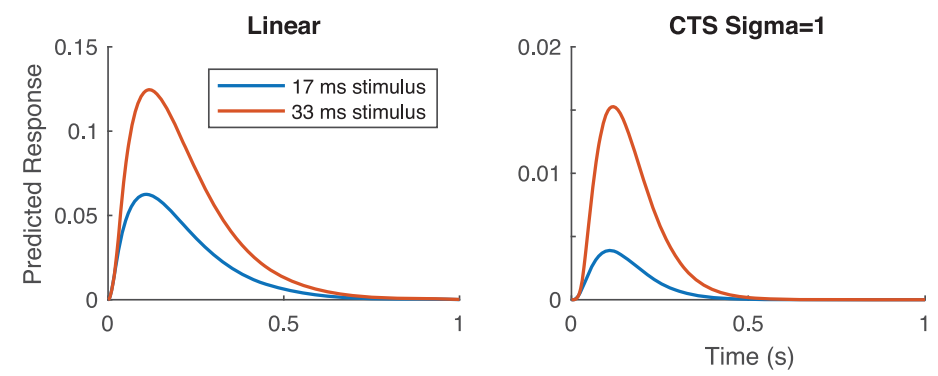

linear predicted time course

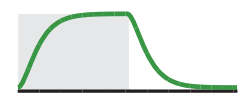

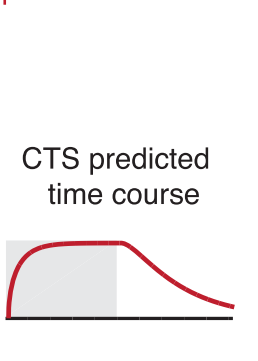

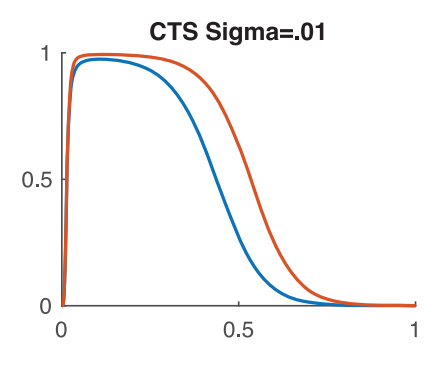

\section{C}

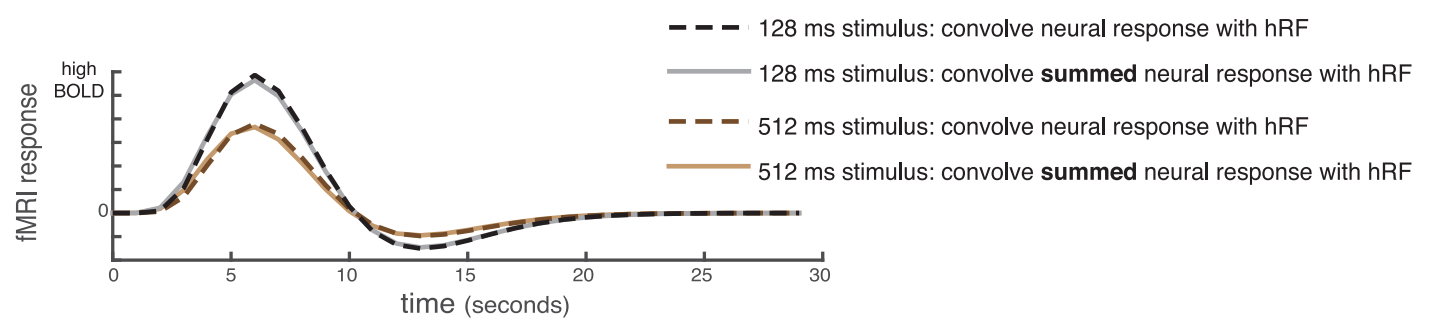

Figure 4. A, The CTS model. The CTS model takes the binary stimulus time course for a trial as input ( 1 whenever the contrast pattern is present, 0 whenever it is absent). The input is convolved with an IRF parameterized by $\tau$ to produce a linear prediction. The second stage is a divisive normalization computation. The numerator is the linear prediction raised point-wise and squared. The denominator is the sum of the squared semisaturation constant, $\sigma^{2}$, and the squared linear response. Finally, the time-varying (TS prediction is summed and scaled $(g)$ to predict the percentage BOLD response. The CTS model was fit for each ROI by finding the values of $\tau, \sigma$, and $g$ that minimized the squared error between the model predictions and the GLM $\beta$ weights. $\boldsymbol{B}$, Predicted neural time series. Left, Predictions from a linear model to a 17 and $33 \mathrm{~ms}$ stimulus, assuming an IRF with time constant $100 \mathrm{~ms}$. The other two panels represent the CTS model predictions with large $\sigma$ (middle) and small $\sigma$ (right). When $\sigma$ is small, the predicted time series are similar for the two stimuli. C, CTS-predicted BOLD time series. (TS model predictions were computed for two stimuli: a $128 \mathrm{~ms}$ and $512 \mathrm{~ms}$ single pulse stimulus (assuming $\tau=0.05 ; \sigma=0.01$ ). The (TS model predictions were then passed through an HRF in one of two ways: either by convolving the (TS model prediction with the HRF (dashed lines) or by convolving the HRF with a single number for each stimulus, the sum of the CTS model prediction (solid lines). For each stimulus, the predicted response to the summed CTS response and to the time-varying CTS response is almost identical. Further, the BOLD response to the longer stimulus is the same shape as the response to the briefer stimulus, just scaled in amplitude.

tor and denominator have similar values, so that the model output is relatively invariant to stimulus duration (hence, more compressive). In contrast, when $\sigma$ is very large, the denominator is approximately a constant, so there is little normalization. In later visual areas (hV4-IPS), $\sigma$ is $\sim 10$ times lower than earlier areas $(\mathrm{V} 1-\mathrm{V} 3 \mathrm{ab})(\sim 0.003$ vs $\sim 0.03$; Fig. $6 \mathrm{~A}$, right $)$, consistent with temporal summation being more sublinear in the modelfree summary (Fig. 3C). The more pronounced sublinearity later in the visual hierarchy is qualitatively similar to the pattern found for spatial summation (Kay et al., 2013a). A consequence of more compressive temporal summation is that the response amplitude varies less with minor changes in stimulus duration, just as greater compression of spatial summation predicts more tolerance to changes in size and position (Kay et al., 2013a). From the current fMRI dataset, there is also a tendency toward shorter time constants $(\tau)$ in earlier areas, with some exceptions (except for VO, V1-V3 have the smallest $\tau, \sim 50 \mathrm{~ms}$; Fig. 6A).

The precision of our parameter estimates in each area depends on the BOLD noise level (the CI of the $\beta$ weights), as well as the specific parameters estimated for that area. To understand how these factors interact, we simulated 1000 datasets for each of three areas: $\mathrm{V} 1, \mathrm{~V} 3 \mathrm{ab}$, and $\mathrm{LO}$. The simulations used the median parameter fits for each area $(\tau$ and $\sigma)$ to generate a noiseless prediction. We then added noise independently for each of the 1000 predictions, according to the noise level in the fMRI measures for that area. Finally, we solved the CTS model for each of the predicted set of responses and analyzed the parameters. This parameter recovery analysis reveals two important results. First, it shows that the parameters for the different areas are distinguishable: models solved from simulations matched to V1, for example, are not confusable with models solved from simulations matched to V3ab or LO (Fig. 6B). Second, the analysis shows that the precision of the parameter estimates differs across areas. For example, for V1, $\tau$ is more precisely specified than $\sigma$, whereas for $\mathrm{LO}, \sigma$ is more precisely specified than $\tau$ (Fig. $6 B$, insets). V3ab is intermediate. These simulations are consistent with the observation that model solutions on the bootstrapped data show a smaller CI for $\tau$ than for $\sigma$ in $\mathrm{V} 1$, and the reverse for LO (Fig. 6A).

To further examine the differences in temporal processing between ROIs, we summarized the CTS model predictions to each ROI 
A
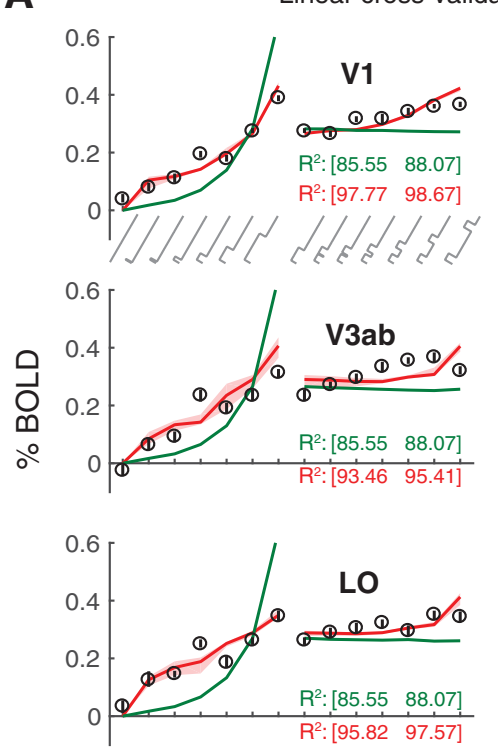
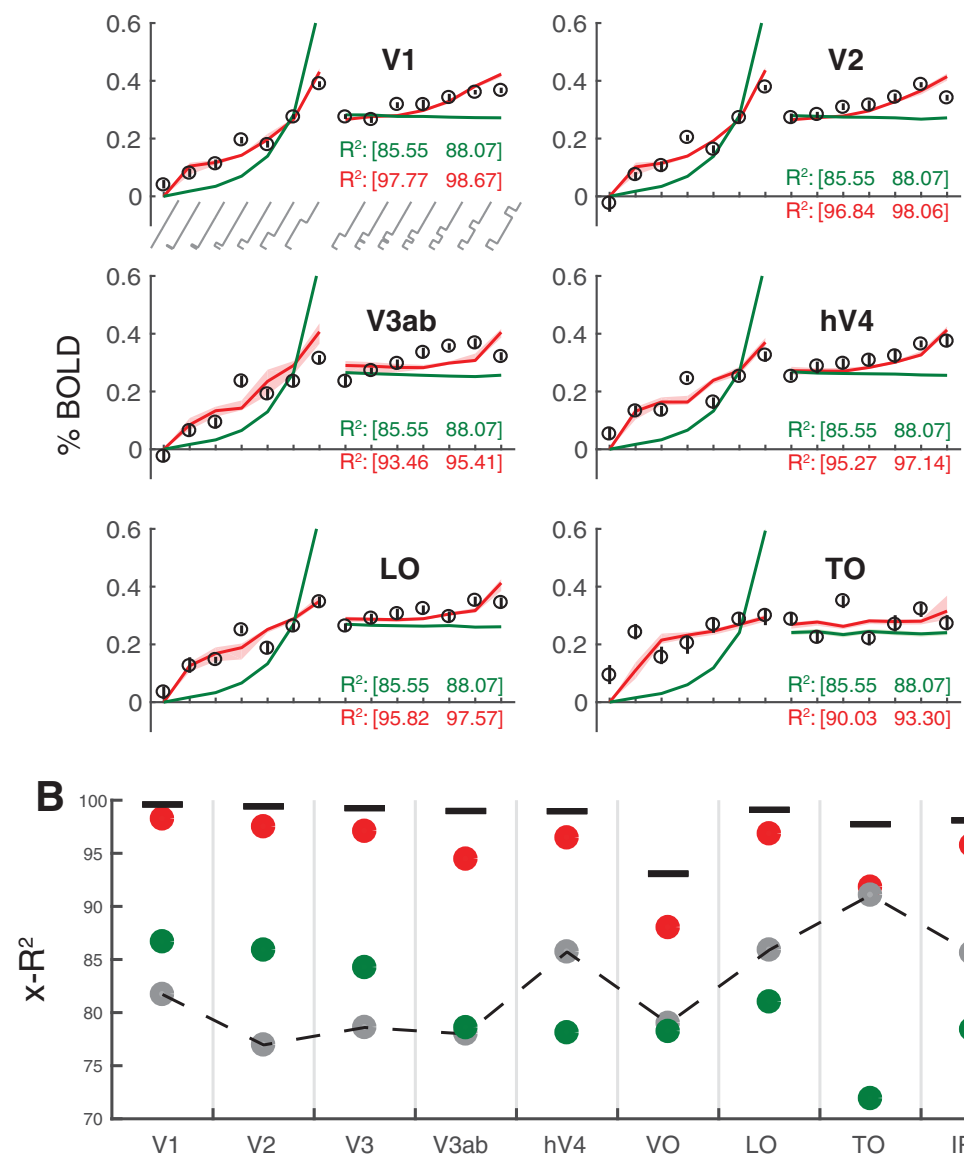

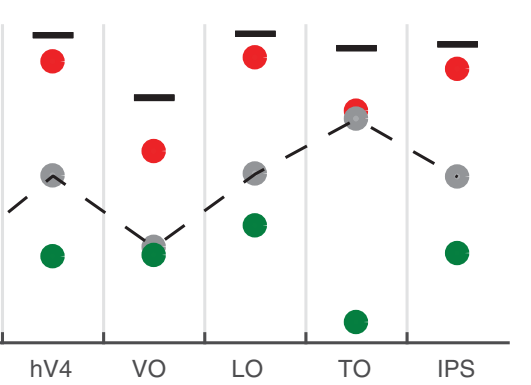

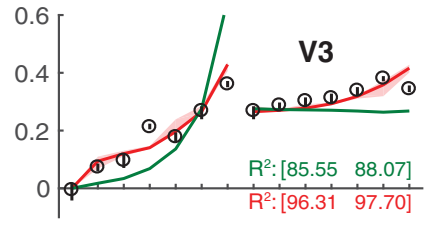
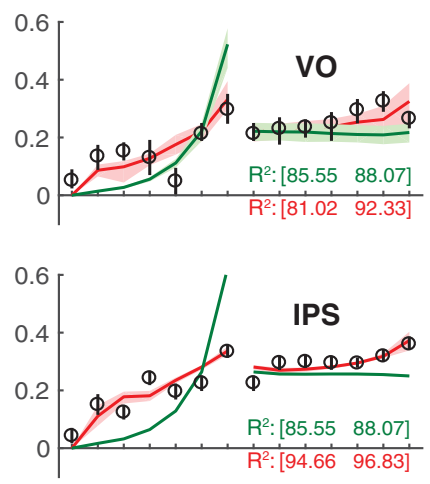

CTS model has higher cross-validated $\mathrm{R}^{2}$ than the linear model in every visual area

median $\mathrm{x}-\mathrm{R}^{2}$ for the CTS model

median $x-R^{2}$ for the linear model

median $x-R^{2}$ for the flat prediction

Figure 5. CTS model fits to BOLD data across visual areas. $A$, Data and predictions. Circles represent BOLD responses to each temporal condition averaged across participants. The temporal conditions on the $x$-axis show increasing durations of one-pulse stimuli ( 0 to $533 \mathrm{~ms}$; left) and increasing ISI of two-pulse stimuli ( 0 to $533 \mathrm{~ms}$, right). Stimulus temporal conditions are as in Figure $2 A$. Error bars indicate the $50 \% \mathrm{Cl}$ bootstrapped across repeated scans. Predictions for the linear (green) and CTS (red) model fits are computed by leave-out-one-condition cross-validation. Shaded regions represent the $50 \% \mathrm{Cl}$ of the predictions across bootstraps (not visible for most of the linear fit because the $\mathrm{Cl}$ is narrow). $\boldsymbol{B}$, The cross-validated accuracy $\left(x-R^{2}\right)$ is higher for the $C \mathrm{CTS}$ model, compared with the linear model in each area. Each circle represents the median cross-validated $R^{2}$ for each model. Error bar indicates the $50 \% \mathrm{Cl}$ across bootstraps. Figure made from the script trf_mkFigure5.m.

response in terms of two metrics that have more directly interpretable units: $\mathrm{R}_{\text {double }}$ and $\mathrm{T}_{\text {ISI }}$ (Fig. $6 \mathrm{C}$ ). $\mathrm{R}_{\text {double }}$ is the ratio between the CTS-predicted BOLD response to a $100 \mathrm{~ms}$ stimulus and a $200 \mathrm{~ms}$ stimulus. Lower $\mathrm{R}_{\text {double }}$ means more compressive temporal summation. Later visual areas have lower $\mathrm{R}_{\text {double }}$ than earlier ones. $\mathrm{T}_{\text {ISI }}$ is the minimal duration separating two $100 \mathrm{~ms}$ pulses such that the response to the paired stimuli is close to the linear prediction from the single stimulus. Similar to previous measurements at longer time scales (Weiner et al., 2010; Mattar et al., 2016), the recovery time is longer for later than earlier visual areas.

\section{The CTS parameters do no vary systematically with} eccentricities from $2^{\circ}$ to $12^{\circ}$

Prior work has shown that temporal encoding in V1 differs between fovea and periphery (Horiguchi et al., 2009). In a separate analysis, we asked whether the CTS model parameters differed as a function of eccentricity. We did not find reliable differences for parafovea $\left(2^{\circ}-5^{\circ}\right)$ versus periphery $\left(5^{\circ}-10^{\circ}\right)$, either in the response amplitude (Fig. $7 A$ ) or in the summary metrics (Fig. $7 B$ ). This may be due to the limited range of eccentricities. Horiguchi et al. (2009) found the biggest difference in temporal sensitivity between fovea and the far periphery $\left(20^{\circ}-60^{\circ}\right)$, with only mini- mal differences between the low-to-mid eccentricity bins we tested.

\section{The CTS model fits replicate across experiments}

We conducted a separate experiment with the identical temporal profiles and two different classes of images: pink noise and faces embedded in pink noise (Fig. 8A). This experiment tests the generalizability across spatial pattern. Because faces were used as one of the textures in this experiment, we included an additional ROI-a face-selective area, which is a combination of the occipital face area and the fusiform face area. A single model was fit to each ROI for each participant, assuming independent gain parameters for the two stimulus classes, and the same time constant and semisaturation constant. The results from the main experiment hold: all visual areas in the second experiment sum sublinearly in time, with the CTS model fitting the data more accurately than the linear model (Fig. 8B). Moreover, as with the main experiment, later areas tended to sum more sublinearly compared with the earlier ones (Fig. 8C). The response amplitudes are slightly lower than those in the main experiment due to stimulus selectivity, and the responses are noisier due to fewer trials per condition; otherwise, the pattern of responses is highly similar. 
A
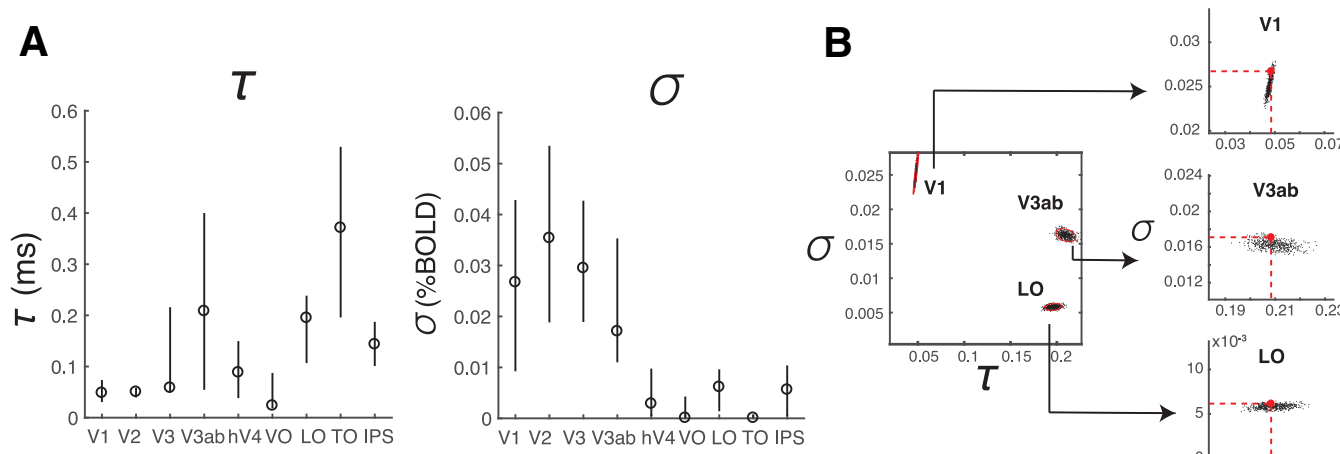

C

$R_{\text {double }}$

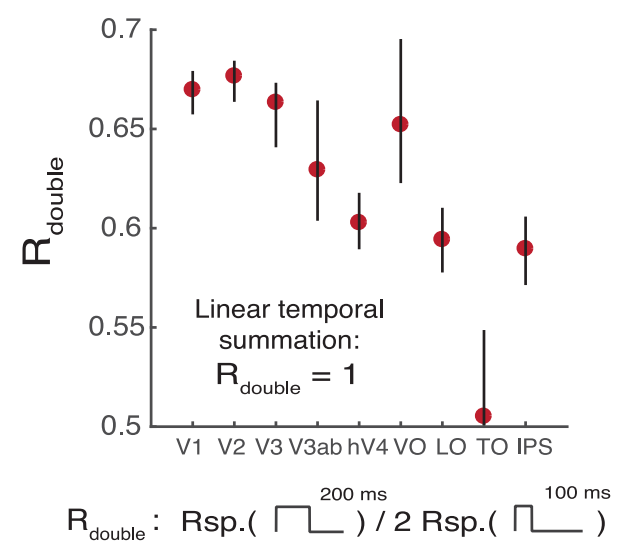

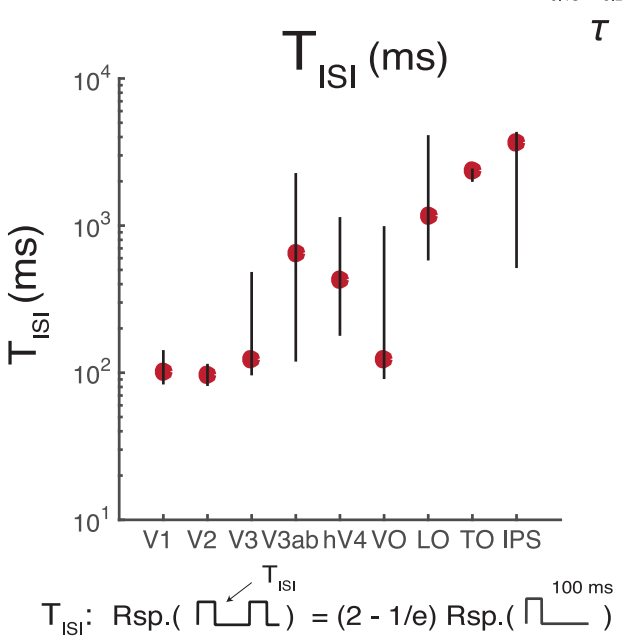

Figure 6. CTS model parameters and summary metrics. $A$, CTS model parameter estimates. The estimated parameter $\sigma$ is smaller for later ( $\sim 0.003$, hV4-IPS), compared with earlier visual areas $(\sim 0.3, \mathrm{~V} 1-\mathrm{V} 3 \mathrm{ab})$, indicating that the temporal sum in the later visual areas deviates more from the linear sum. The time constant $\tau$ is short in V1-V3, compared with most of the later visual areas. $B$, CTS parameter recovery. The precision with which parameters can be fit depends on the noise level in the data and the specific parameter values. We simulated data using the median $\tau$ and $\sigma$ from V1, V3ab, and L0, and the noise levels estimated from these areas. The analysis shows that, under these conditions, $\tau(x$-axes) is specified most precisely in V1 and least precisely in V3ab; the opposite pattern is found for $\sigma$ (y-axes). C, Summary metrics. Two summary metrics of the CTS model reveal a pattern across ROls. $R_{\text {double }}$ is the ratio of the predicted response to a $200 \mathrm{~ms}$ pulse divided by twice the response to a $100 \mathrm{~ms}$ pulse. $R_{\text {double }}$ is $<1$ for all ROls, indicating subadditivity, and decreases along the visual hierarchy (V1-V3, $\left.\sim 0.67, L 0-I P S,<0.6\right)$. $\mathrm{T}_{\mid \mathrm{ISI}}$ is the length of ISI required for the response to two $100 \mathrm{~ms}$ pulses to approach the linear prediction. $\mathrm{T}_{\text {ISI }}$ is short in the earlier areas (V1-V3, $100 \mathrm{~ms}$ ) compared with most of the later areas. Figure made from the script trf_mkFigure6.m.

\section{Differences in parameters across ROIs are not explained by differences in HRFs}

We found that the CTS parameters representing temporal processing differed systematically along the visual hierarchy, with a tendency toward a pronounced nonlinearity and longer time constant in later visual areas. These results were obtained with a model in which a single HRF was fitted to each individual subject, but not to each ROI separately. Fitting a single HRF to each area is robust in that it reduces sensitivity to noise within an area as signal. However, if the actual HRF differs systematically across areas, then it is possible that enforcing a single HRF will result in biased estimates of the $\beta$ weights. In this section, we consider the possibility that the differences in derived metrics $\left(R_{\text {double }}\right.$ and $\mathrm{T}_{\text {ISI }}$ ) across ROIs might be explained by variations in the HRF rather than differences in the underlying neuronal responses.

To address this question, we estimated a separate HRF for each ROI and each subject. We estimated the ROI-specific HRF in two ways: from the retinotopy experiment and from the first temporal experiment. For each area, the HRFs were parameterized as a difference of two gamma functions (Friston et al., 1998; Worsley et al., 2002). The resulting HRFs were broadly similar across ROIs. For example, the time course of the HRF of an intermediate area, $\mathrm{hV} 4$, is within $1 \mathrm{SD}$ of the time course of all other ROIs at almost all time points from both retinotopy and the temporal experiment (Fig. 9A,B). There are some qualitative differences, such as a larger poststimulus undershoot in later areas, particularly as estimated by the temporal experiment (VO, LO, TO, IPS). To assess the impact of these modest differences in the HRFs across areas, we recomputed the CTS model parameters and the two derived summary metrics, $\mathrm{R}_{\text {double }}$ and $\mathrm{T}_{\text {ISI }}$ (Fig. 9 C). The general pattern of results is the same whether the HRFs are fit to each area or to each individual: $\mathrm{R}_{\text {double }}$ tends to decrease along the visual hierarchy, and $\mathrm{T}_{\text {ISI }}$ increases.

\section{The CTS model is more accurate than a TTC model in later visual areas}

The CTS model was implemented to capture subadditive temporal summation using canonical neural computations (filtering, exponentiation, normalization). An alternative model, in which neuronal responses are thought to reflect the outputs of TTC, has been proposed to account for psychophysical temporal sensitivity (Watson and Robson, 1981; Hess and Plant, 1985; Watson, 1986) and fMRI responses in V1 (Horiguchi et al., 2009) and extrastriate cortex (Stigliani et al., 2017). This TTC model linearly combines the output from a sustained and a transient temporal frequency channel. The sustained channel has a mostly positive IRF and is linear and the transient channel has a balanced (zerosum) IRF and its output is squared (Fig. 10A). The TTC model 
A

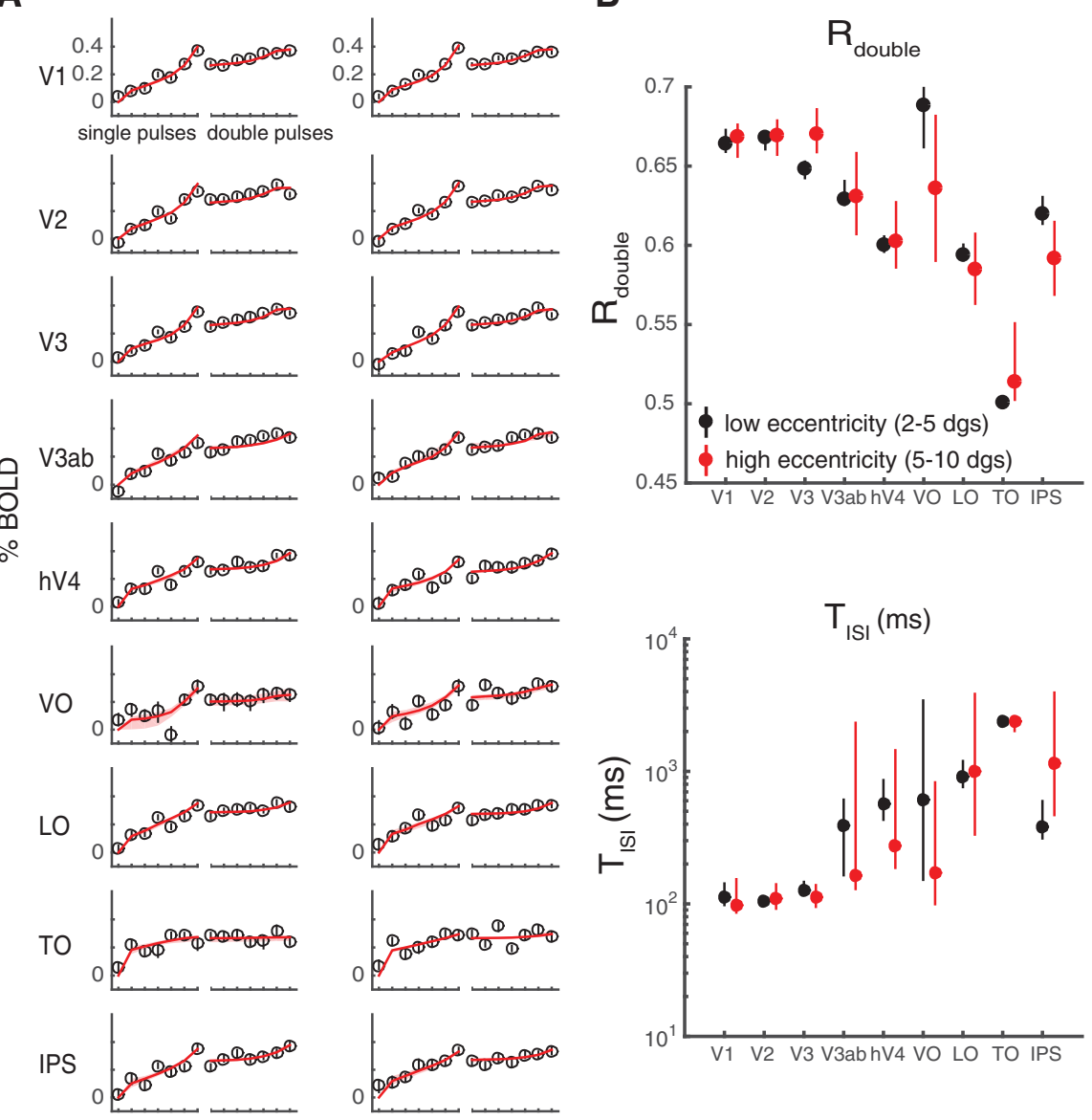

Figure 7. CTS model fits by eccentricity. Data from the main fMRI experiment are replotted, separating each ROI into two eccentricity bins. $A$, CTS model fit to low and high eccentricity bins. Left panels, Data and CTS model fits restricted to voxels with pRF centers within $2^{\circ}-5^{\circ}$. Right panels, Data and CTS model fits restricted to voxels within $5^{\circ}-10^{\circ}$ eccentricity. $\boldsymbol{B}$, Summarized metrics for different eccentricity bins. The summarized metrics do not differ systematically between the two eccentricity ranges. Each dot indicates the median of the metrics summarized for 100 bootstraps of data (across scans). Error bars indicate $50 \%$ Cl. Figure made from the script trf_mkFigure7.m.

contains filtering and exponentiation but not normalization. The specific forms of the IRFs in the TTC model are derived from psychophysics, not neural data, and hence are assumed to be the same in all visual areas; the model is fit only by varying the relative weights of the two channels.

We fit the TTC model to the bootstrapped $\beta$ weights estimated from the first temporal experiment, and compared this with the CTS model fits. In early visual areas (V1-V3), the two models have similar accuracy (as assessed by cross-validated $R^{2}$ ). In later visual areas (e.g., in LO, TO, and IPS), the CTS model captures the data better. For the later areas, the TTC model systematically underpredicts $\beta$ weights for the one-pulse conditions and overpredicts the two-pulse conditions (Fig. 10 B,C). Because of the relatively brief time scales of the IRFs in the TTC model, the predicted response to the second of two pulses is largely unaffected by the first pulse. This will result in an overprediction for any visual area with long temporal windows. As noted earlier, measurements further in the periphery of V1 have greater sensitivity to stimulus transients (Horiguchi et al., 2009); it is therefore likely that, had our measurements extended into the far periphery, the CTS model would need to be augmented with a second, transient channel.

Although the predictions from the two model forms differ in detail, there are some important commonalities. For example, both the TTC model and the CTS model show a compressive function (subadditivity) with stimulus duration, which matches the trend in the data. Further, both models show a sensitivity to ISI, with a greater response predicted for longer ISIs, qualitatively matching the data, and consistent with short-time-scale adaptation (and recovery from adaptation). For these reasons, both models are more accurate than the linear model, which does not capture either of these trends in the data. The TTC model is sometimes linked to two pathways emerging from the retina (parvo- and magno-pathways) (Rodieck et al., 1985; Wandell, 1995). However, both models are proposed at the computational rather than implementational level (Marr, 1982; Carandini and Heeger, 2011; Kay, 2017), and we remain agnostic as to whether the temporal compression observed in the data and predicted from the models arises from retinal ganglion cells, normalization within visual cortex, feedback from higher areas, or a combination of these.

\section{Alternative implementations of CTS model}

The nonlinear component of the CTS model was implemented as a divisive normalization. This model fit the data much more accurately than a linear model, and divisive normalization is a good descriptor of a wide range of neural phenomena (Carandini and Heeger, 2011). However, there are many choices of static nonlinearities. In prior work, a power law static nonlinearity was used to model compressive spatial summation in fMRI (Kay et al., 2013a; Winawer et al., 2013). Although the form of the two nonlinearities differ, we found that refitting the fMRI data with the power-law nonlinearity produced results that were highly similar to the fits with the divisive normalization implementation (Fig. 11). The model accuracy was not distinguishable when using a power law versus divisive normalization, and the two derived metrics, $\mathrm{R}_{\text {double }}$ and $\mathrm{T}_{\mathrm{isi}}$, showed the same pattern. Hence, the fMRI data in these experiments do not distinguish the two forms of the compressive nonlinearity. The power-law nonlinearity has the advantage of ease of interpretation; the exponent indicates how much the response deviates from linear. Divisive normalization has the advantage of strong support from many neural systems (Carandini and Heeger, 2011). It might be possible to distinguish the two by measuring responses to stimuli with very brief durations or very low contrasts. One difference between the two nonlinearities is the precision in which parameters are recovered. For example, $\tau$ is recovered with high precision for most visual areas in the divisive normalization implementation; for the power-law implementation, the exponent $\varepsilon$ is recovered more accurately than $\tau$ (simulations not shown).

Finally, for completeness, we fit two other variants of the CTS model: one in which the exponent of 2 in the numerator and denominator was replaced by a free parameter, $n$, as follows: 
A
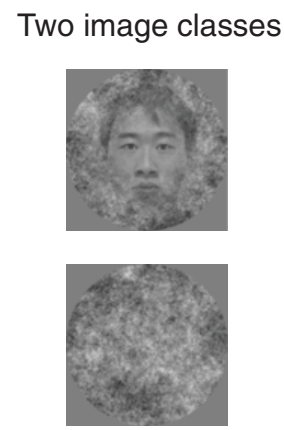

(24 dg. diameter)
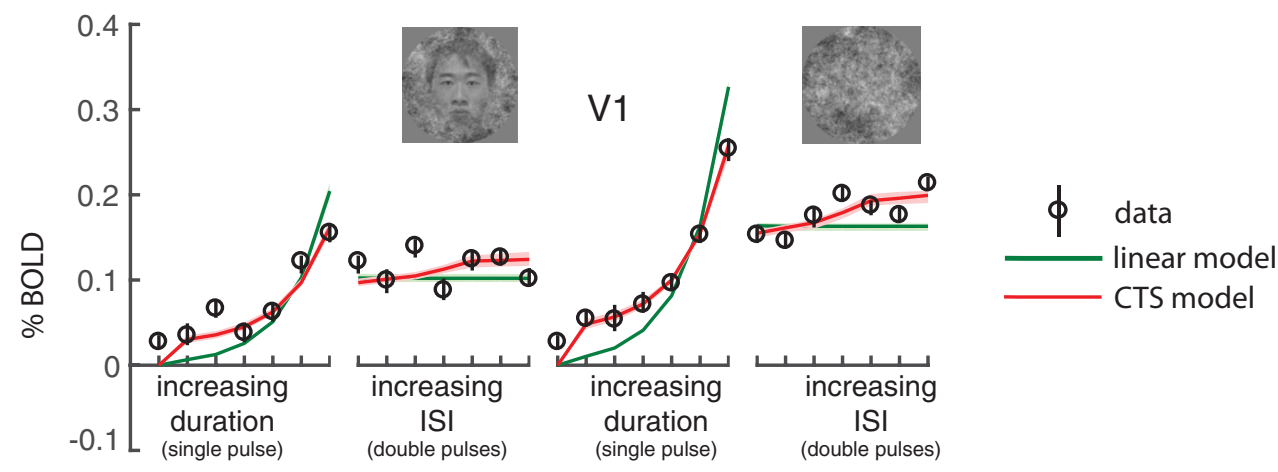

B
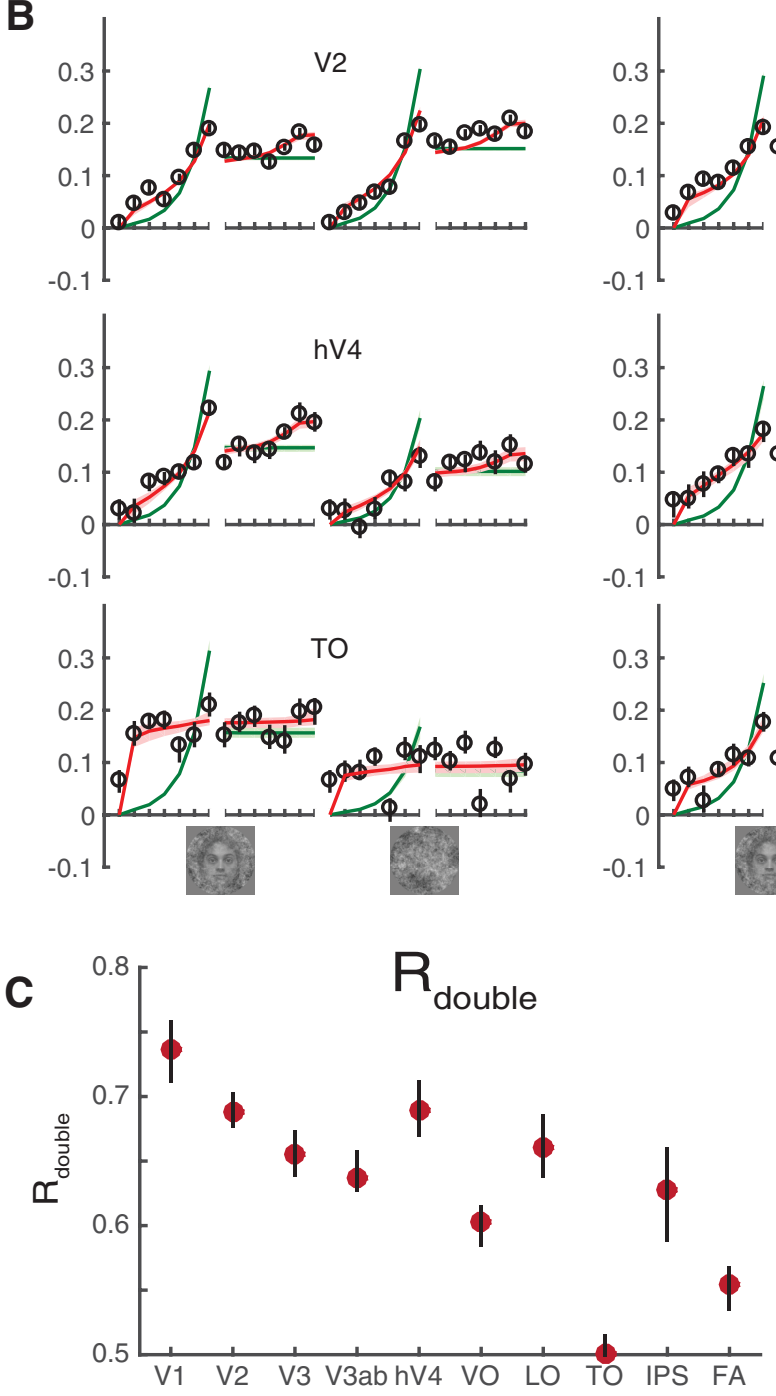
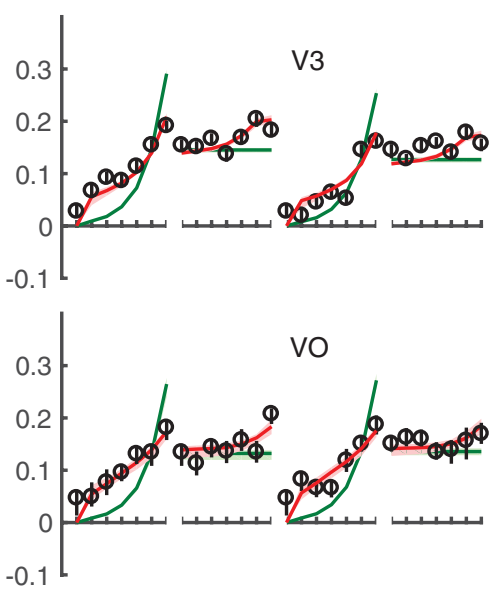

IPS
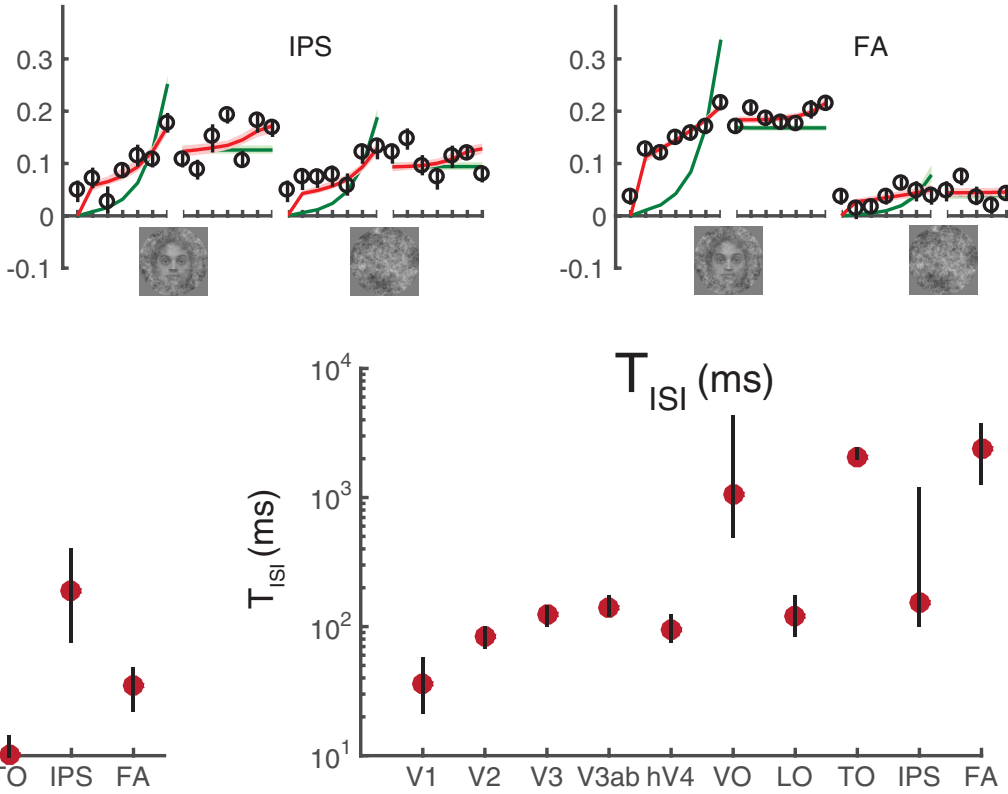
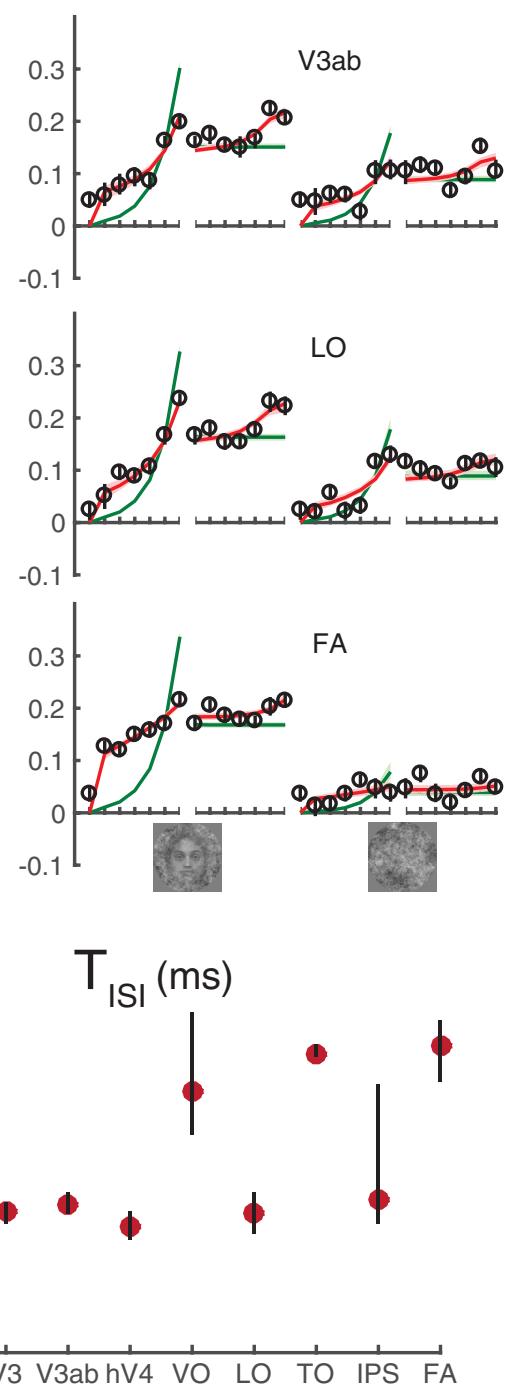

Figure 8. fMRI data and model fits from a second experiment. A, Stimuli and V1 responses. The two stimulus classes (noise patterns and faces embedded in noise patterns) were randomly interleaved within runs. Temporal conditions were identical to those in Figure 4. The general pattern of responses and model fits are highly similar to those in the main experiment, with the CTS model fitting the data much more accurately than the linear model. B, CTS model fit to extrastriate visual areas. The CTS model (red) fit the data more accurately than the linear model in all visual areas. $C$, Parameters derived using the CTS model fit. The derived metrics, $R_{\text {double }}$ and $\mathrm{T}_{\mid \mathrm{SI} /}$, show similar patterns as in the main experiment: decreased $\mathrm{R}_{\text {double }}$ and increased $\mathrm{T}_{\mid \mathrm{IS}}$ in higher visual areas. Figure made from the script trf_mkFigure8.m.

$$
B O L D_{C T S}=g \sum \frac{x^{n}}{\sigma^{n}+x^{n}}+e
$$

and one in which the exponents in the numerator and denominator were fit with separate free parameters, $m$ and $n$ as follows: 
A
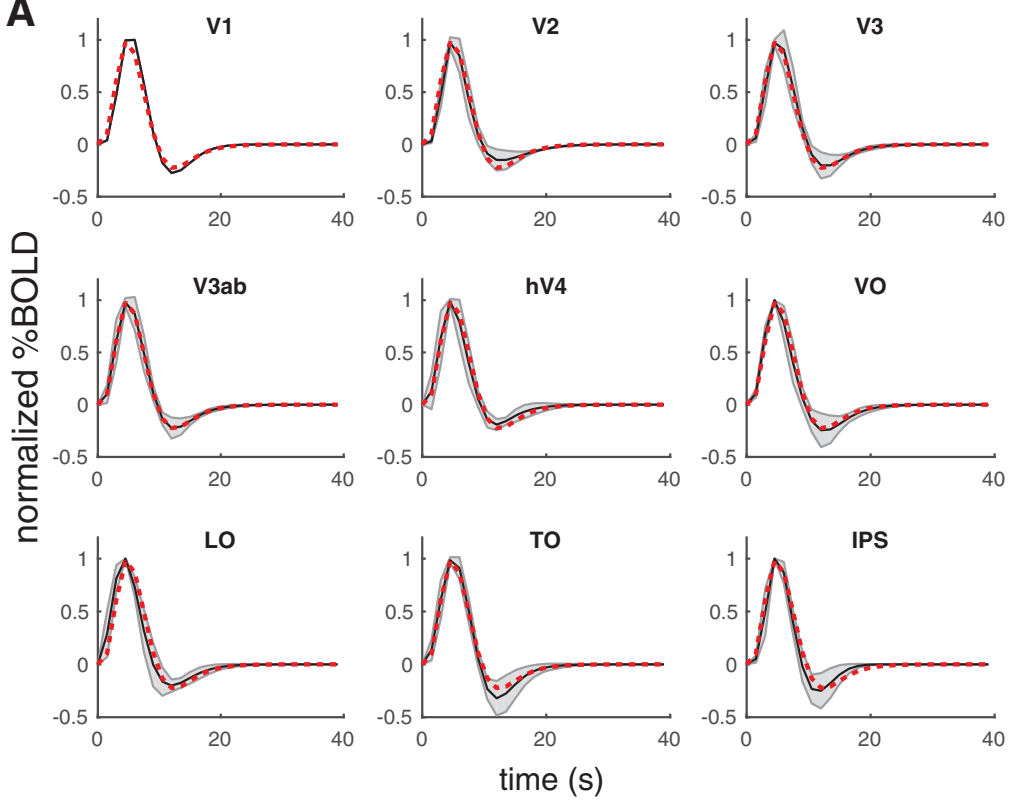

B
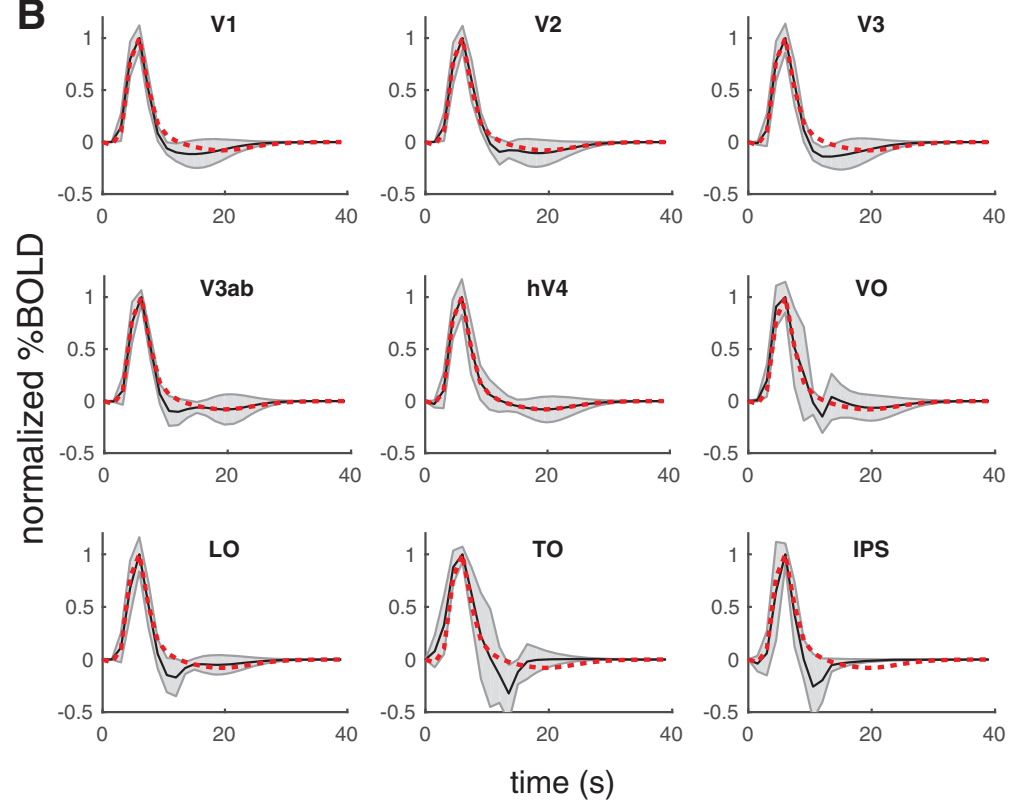

C

$R_{\text {double }}$

$\mathrm{T}_{\mid \mathrm{SI}}(\mathrm{ms})$
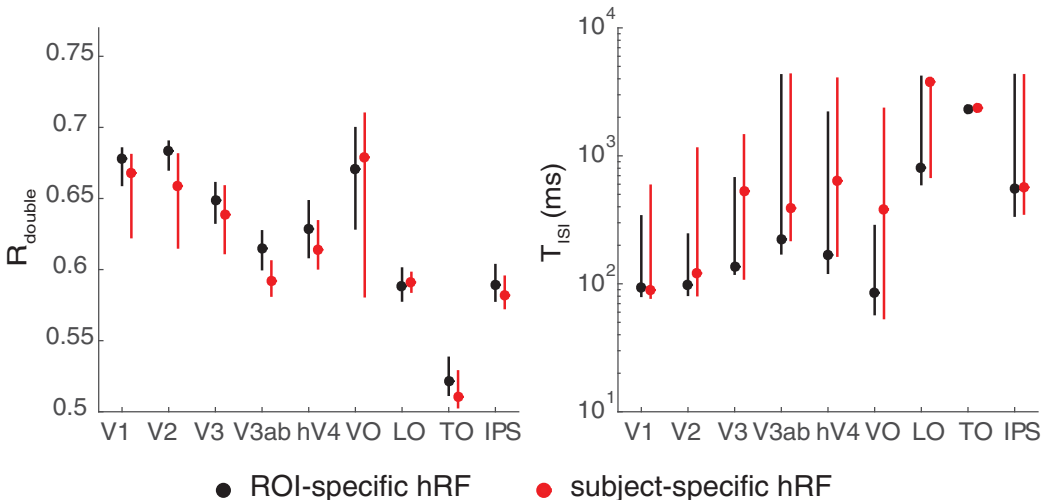

- subject-specific hRF

Figure 9. HRFs for individual ROIs. A, HRFs derived from the retinotopy experiment. Shaded black curves indicate the estimated HRF from each ROI (mean and SD across subjects). Dotted red line indicates the mean HRF estimated for hV4, replotted in each panel for comparison. The hV4 HRF does not differ much from the other individually estimated HRFs. $\boldsymbol{B}$, Same as $\boldsymbol{A}$, but the HRFs are

For the implementations with more free parameters, the multiple nonlinear parameters ( $\sigma$ and exponents) appear to trade off to a certain degree, so that the error bars on the separate parameters tend to be larger than those for the simpler implementation of the CTS model. Moreover, as the models become more complex, the separate parameters are harder to interpret. For example, $\sigma$ is an order of magnitude bigger in the rightmost compared with the leftmost model, but it is also raised to a higher exponent $(n)$ in the rightmost model; hence, the effect of normalization may be similar for the two models (Fig. 12A).

Because the individual model parameters are difficult to interpret, and the models differ in the number of free parameters, it is most informative to compare them on summary metrics. This shows that the same general pattern holds for all implementations: early visual areas (V1-V3) tend to have shorter $\mathrm{T}_{\mathrm{isi}}$ and larger $\mathrm{R}_{\text {double }}$ (Fig. 12B). Area TO is at the opposite extreme, with long $\mathrm{T}_{\text {isi }}$ and low $\mathrm{R}_{\text {double }}$. All three model variants have very high cross-validated accuracy, substantially outperforming the linear model (Fig. 12C). Although the models with more parameters have numerically higher accuracy, the difference is small; hence, we tend to favor the simpler, more interpretable implementation. This bias toward simpler implementations is consistent with other uses of divisive normalization (Carandini and Heeger, 2011).

\section{Discussion}

\section{Summation and adaptation in visual cortex}

We report that temporal summation is subadditive throughout human visual cortex. Across 10 visual areas, BOLD responses to long stimuli were less than the linear prediction from briefer stimuli. This subadditivity was especially pronounced in areas anterior to V1-V3. We captured this effect with a new temporal receptive field model, comprised of a linear stage followed by a static nonlinearity. This compressive temporal summation model made highly accurate predictions for the fMRI data, and in all visual areas was substantially more accurate than a linear model. A single model accurately predicted two phenomena: subadditiv-

derived from the temporal experiment. C, CTS summary metrics $\left(R_{\text {double }}\right.$ or $\left.T_{\mid S I}\right)$ derived from the CTS model, fit either with subject- and ROI-specific HRFs (black) or only subject-specific ROls (red). Figures made from the script trf_mkFigure9.m. 
A

Two-temporal-channels
model schematics
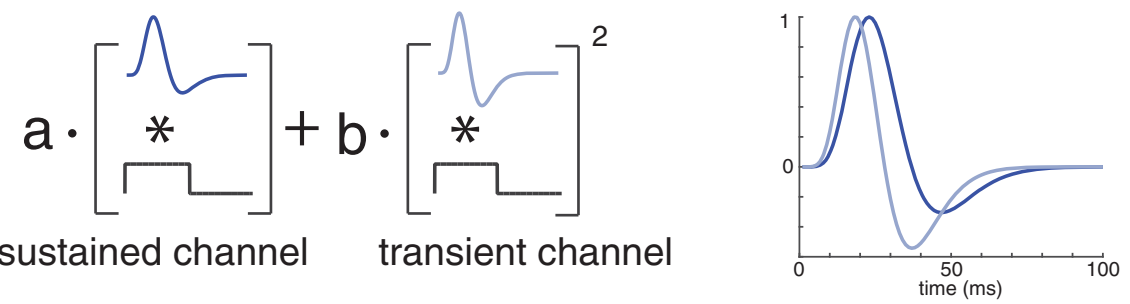

B

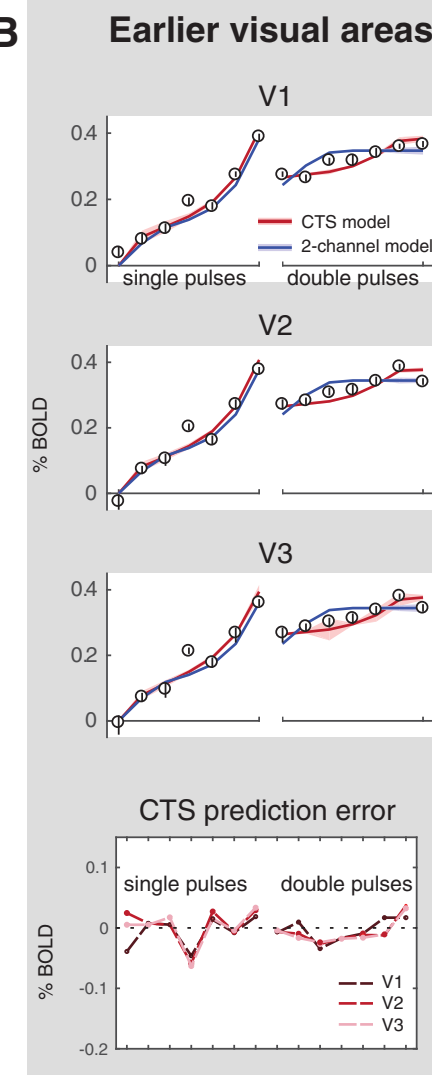

Two-channel prediction error

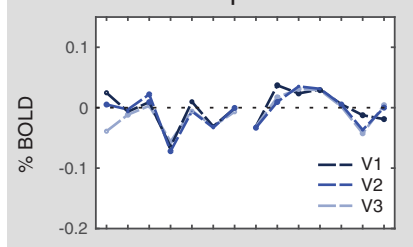

Later visual areas

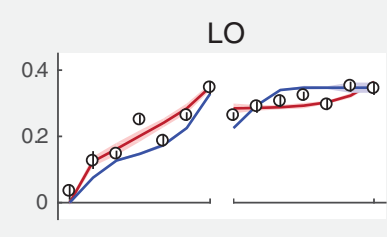

TO

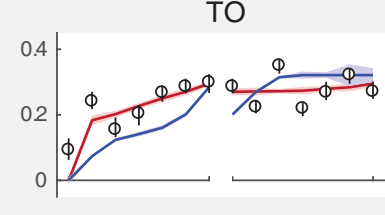

IPS

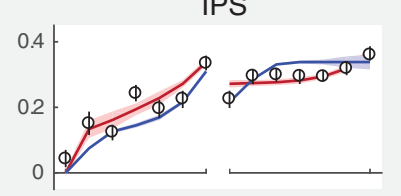

CTS prediction error

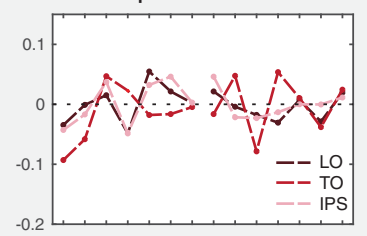

Two-channel prediction error

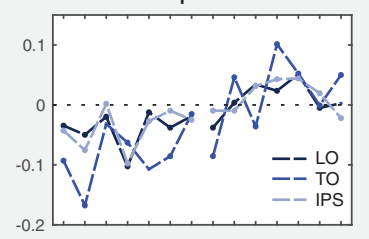

C

cross-validated $\mathrm{R}^{2}$

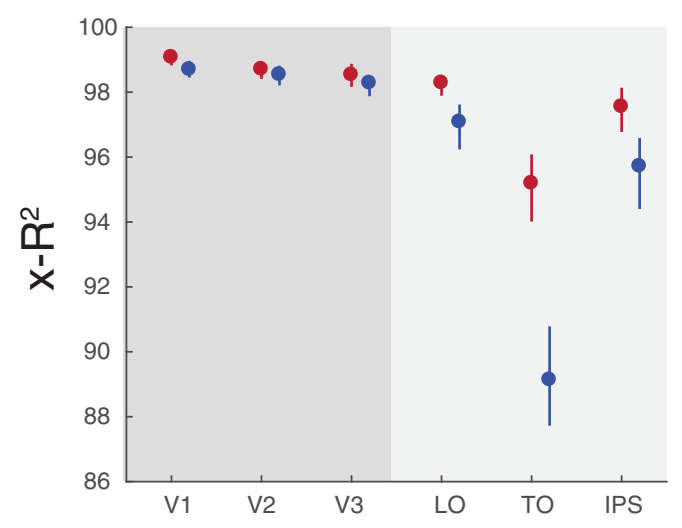

- CTS

2-channel model

\section{D transient weight/sustained weight (b/a)}

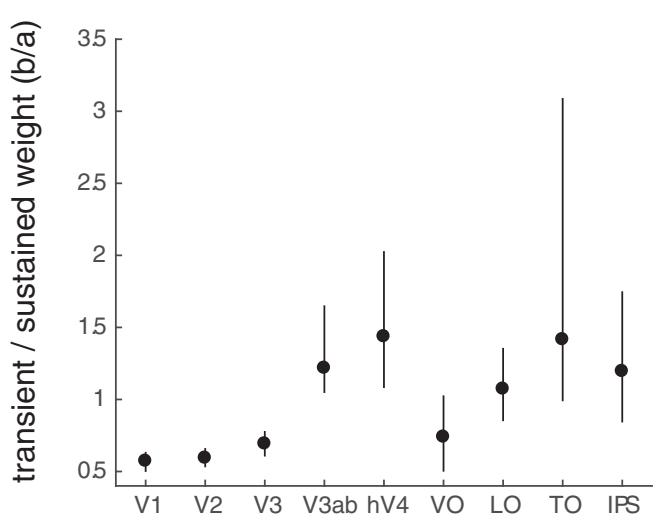

Figure 10. The CTS model describes data in later visual areas better than the TTC model. $A$, TTC model schematic. The model combines the outputs (convolution of stimulus and channel-specific IRF) from a sustained channel (mostly positive IRF, linear output) and a transient channel (biphasic IRF, squared output). This model was used by Horiguchi et al. (2009) to fit fMRI data to temporal modulations in luminance. The fMRI implementation was adapted from previous models that account for psychophysical data (Watson, 1986). B, The CTS and TTC model fit the data about equally well in V1-V3, whereas the CTS model fits the data better in later areas (LO, TO, and IPS). In the later visual areas, the TTC model systematically underpredicts the response to the one-pulse stimulus and overpredicts the response to two-pulse stimuli (bottom four panels). $\boldsymbol{C}$, The cross-validated accuracy $\left(x-R^{2}\right)$ is higher for the CTS model in later areas. $D$, The predicted weights for the sustained versus the transient channels differ across areas. The ratio between the weights for the transient versus for the sustained channels is plotted for each visual area (median, and $50 \% \mathrm{Cl}$ across bootstraps). Later visual areas tend to show increasingly high transient weights, consistent with Stigliani et al. (2017). Figure made from the script trf_mkFigure10.m.

ity in the duration-response function and adaptation over short time scales (ISIs ranging from 0 to $528 \mathrm{~ms}$ ). This indicates that both effects, the subadditivity with respect to duration and the response reduction to repeated stimuli, may arise from the same underlying processes.

A wide range of prior experimental measures are consistent with temporal subadditivities in visual cortex. For example, at the scale of 3-24s, the fMRI response in V1 to a long presentation of a reversing contrast pattern is less than the prediction from a shorter presentation (Boynton et al., 1996); the fMRI response to repeated contrast patterns is larger for $1 \mathrm{~s}$ ISIs than $3 \mathrm{~s}$ ISIs (Heckman et al., 2007); the response of a V1 neuron to a steady flash is not predicted by its temporal frequency tuning and decreases over time (Tolhurst et al., 1980); the response of a neuron to a repeated stimulus is less than the response to the first stimulus (Priebe et al., 2002; Motter, 2006). Here we both quantified temporal subadditivities across the cortical visual hierarchy and account for the effects with a forward model. The model generalizes 
A Compute linear Rsp.

( $\tau)$

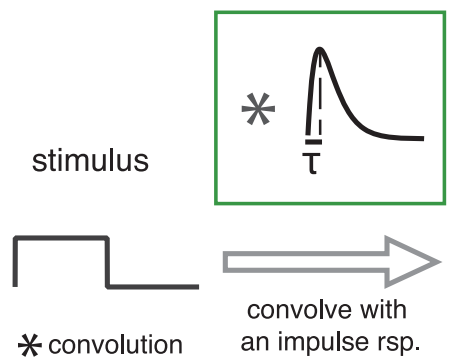

Compute CTS Rsp.

$(\varepsilon)$

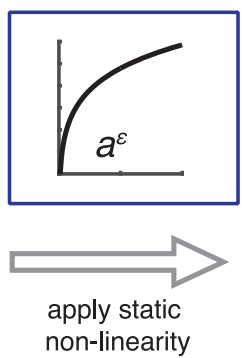

convert to fMRI

(g)

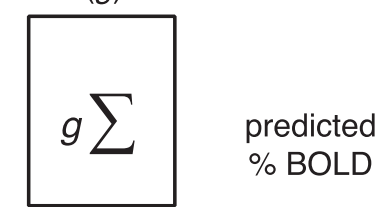

linear predicted time course

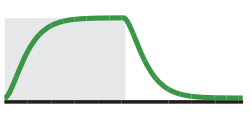

B
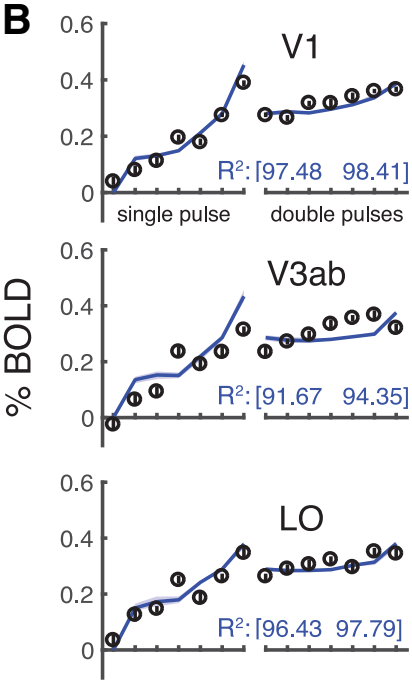
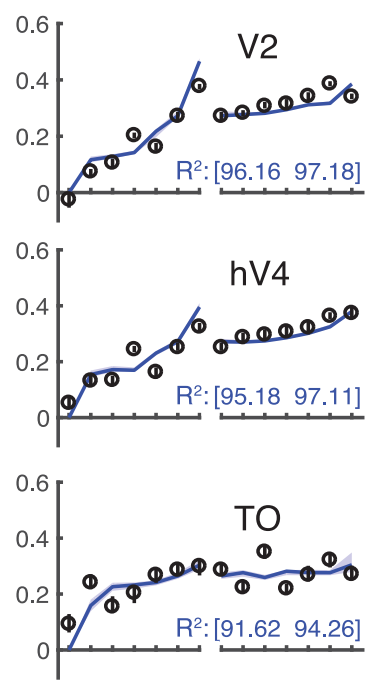

$\phi$ data - CTS prediction (power-law implementation)
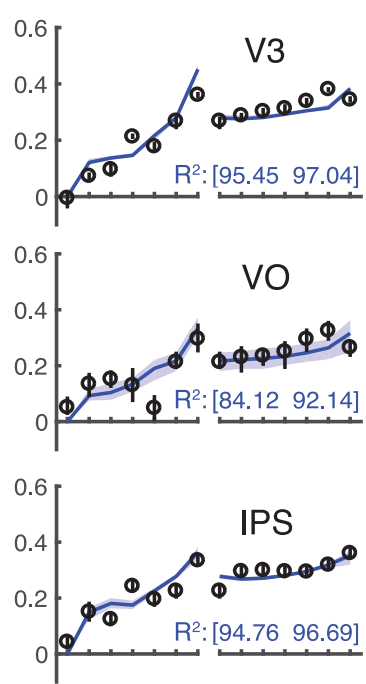

C

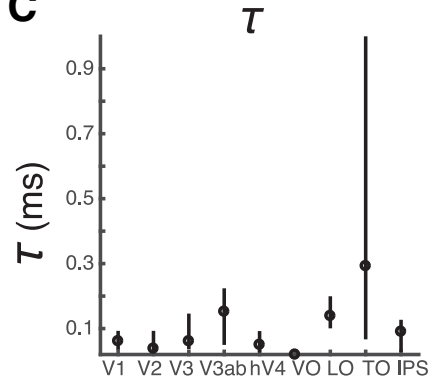

scale and sum the rsp. time course
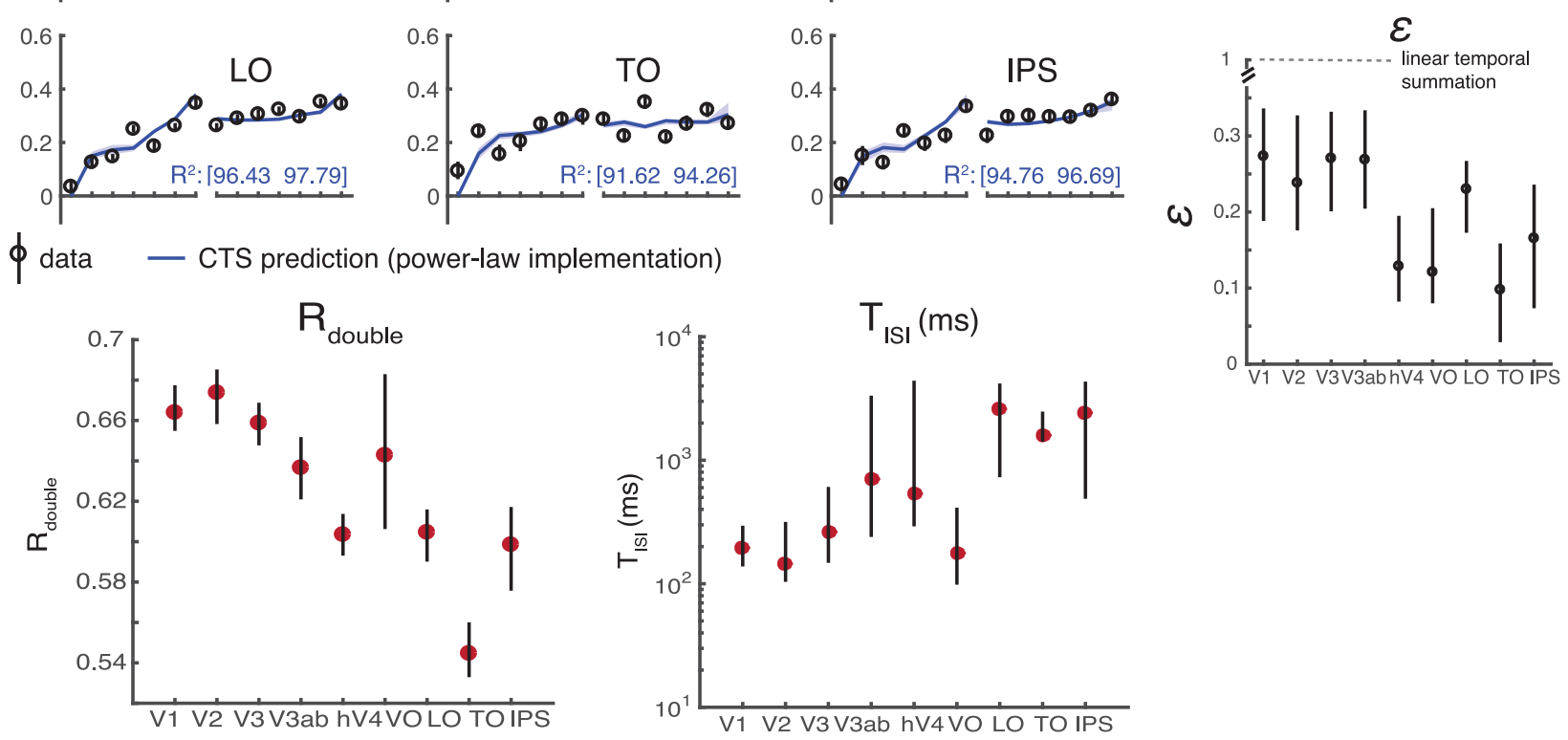

Figure 11. CTS model implemented with a power-law nonlinearity. $\boldsymbol{A}$, Model description. The model is identical to that shown in Figure 4, except that the static nonlinearity is a power-law (parameterized by $\varepsilon$ ) rather than a divisive normalization. If $\varepsilon$ is 1 , the model is linear; and if $\varepsilon$ is $<1$, it is compressive. $\boldsymbol{B}$, (ross-validated model fit to the data from the main fMRl experiment. The model describes the data with high accuracy (for plotting conventions, see Fig. $5 A$ ). $C$, Model parameters. The estimated exponent $\varepsilon$ is $<1$ in each area, and is lower (more sublinear) in later areas $\left(\sim 0.15\right.$, hV4-IPS vs $\sim 0.25$, V1-V3ab). The time constant $\tau$ is similar to that observed for the normalization fit. $\boldsymbol{D}$, Summary metrics. $R_{\text {double }}$ is $<1$ for all R0ls, indicating subadditivity, and decreases along the visual hierarchy (V1-V3, 0.67, L0-IPS, < 0.62). $\mathrm{T}_{\mathrm{ISI}}$ is short in the earlier areas (V1-V3, $\sim 100 \mathrm{~ms}$ ) compared with most of the later areas. Figure made from the script trf_mkFigure11.m.

from the observed effects, as it takes arbitrary temporal patterns as inputs. The two operations (linear summation and a compressive nonlinearity) provide a simple and interpretable set of computations that can be used to characterize neural response properties across visual areas. For example, an implication of the $\mathrm{T}_{\text {ISI }}$ measures is that, when designing an fMRI experiment, stimuli must be spaced by at least $100 \mathrm{~ms}$ to avoid significant interactions in V1 responses, and at least $1 \mathrm{~s}$ in TO or IPS.

\section{Subadditivities in fMRI}

In principle, the subadditivity could arise from the neuronal responses, coupling between neuronal processes and the BOLD signal, or a combination of both. There are several reasons to believe that at least a significant part of the observed nonlinearity is neuronal in origin. First, single-unit measurements of cortical neurons show temporal subadditivities (Tolhurst et al., 1980; Motter, 2006), and it is more parsimonious to attribute subadditivities in the single-unit and BOLD measurements to a single cause. Second, we find greater subadditivities in later than earlier visual areas, consistent with a cascade architecture in which later areas add additional nonlinearities to the outputs from earlier areas (Heeger et al., 1996; Simoncelli and Heeger, 1998; DiCarlo et al., 2012; Kay et al., 2013a, b); in contrast, there is no reason to expect that the coupling between neuronal signals and the hemo- 

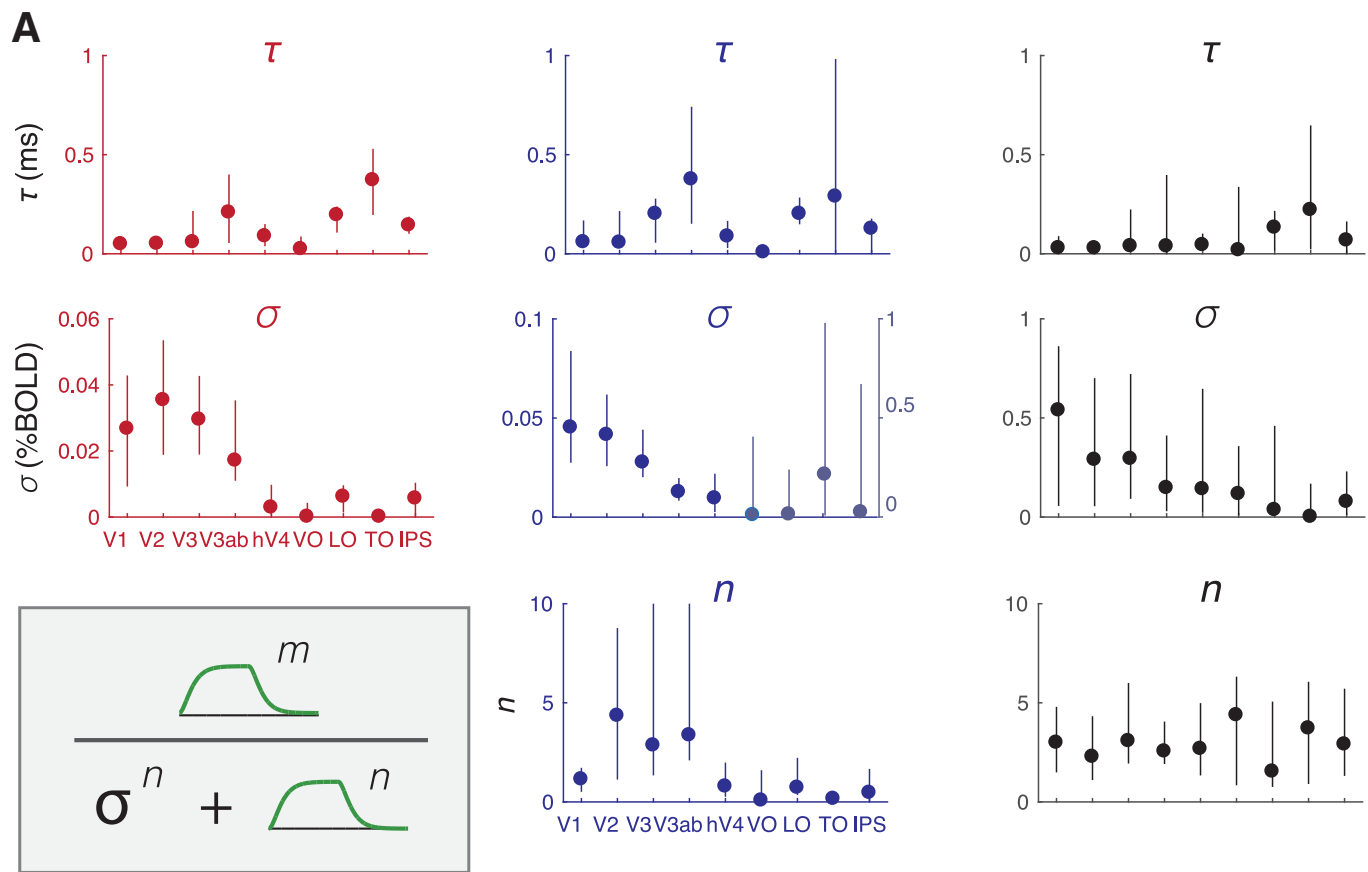

case 1: $m=n=2$;

case 2: $m=n$; and the value of $n$ varies

case 3: $m$ and $n$ vary independently;

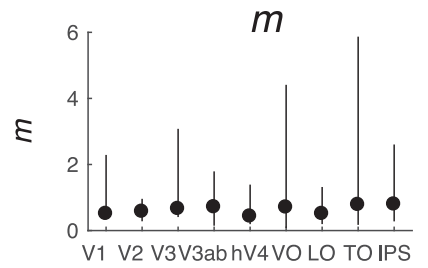

B
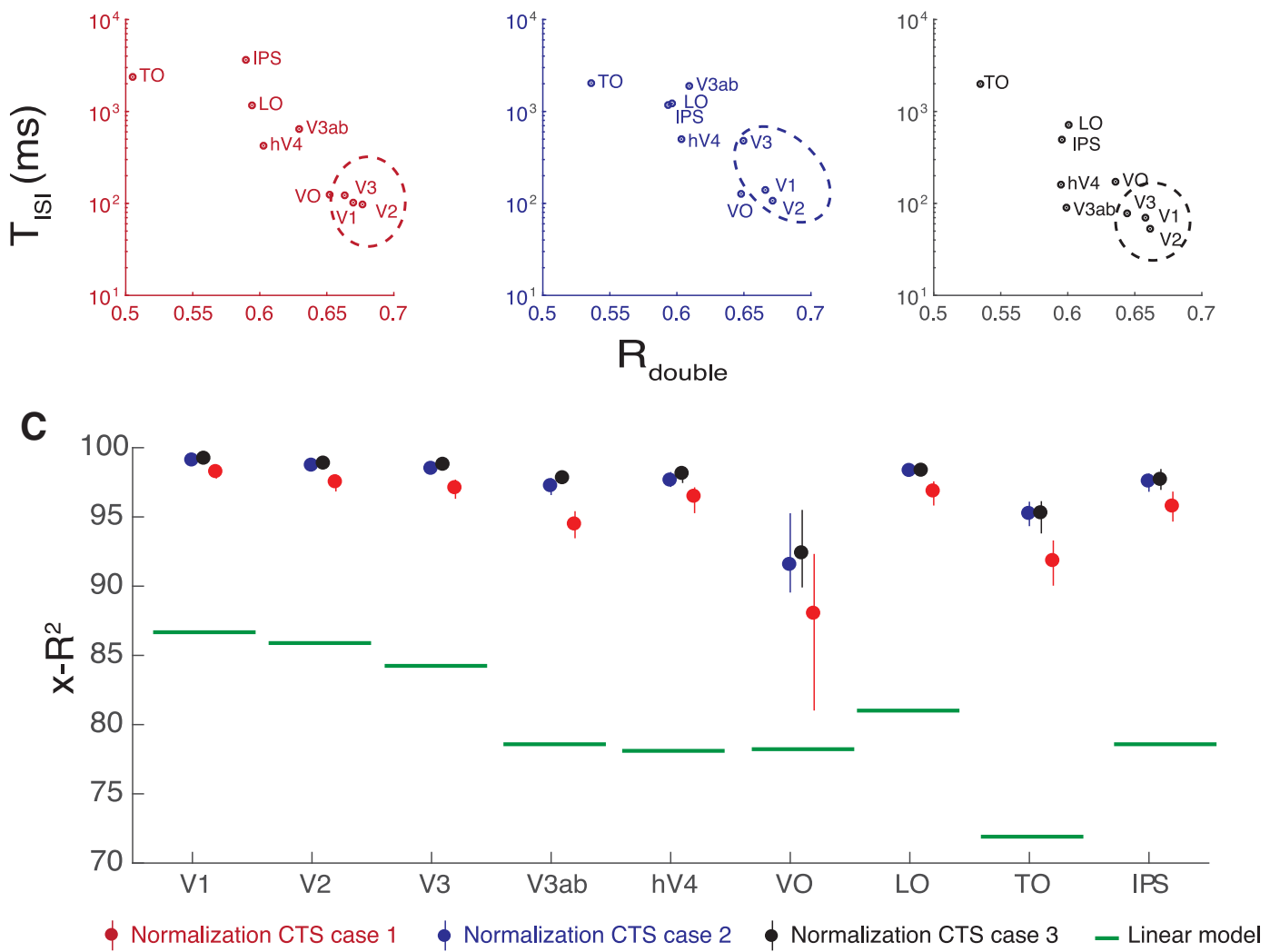

Figure 12. Three implementations of divisive normalization for the CTS model show the same pattern of effects. $\boldsymbol{A}$, We show three implementations of divisive normalization, with increasing numbers of free parameters from left to right. Left, The simplest implementation (same as Figs. 4-10), with the exponents fixed at 2. Middle, The exponent is a free parameter. Right, The exponents in the numerator and denominator are each free parameters. In each case, the normalization step is preceded by normalization with an IRF (Figure legend continues.) 
A
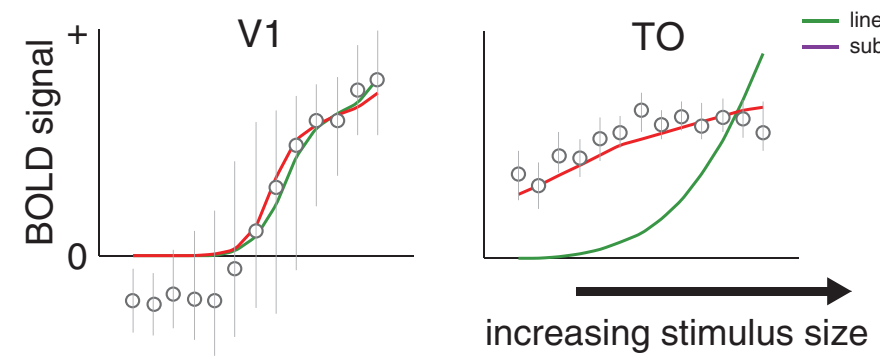

B

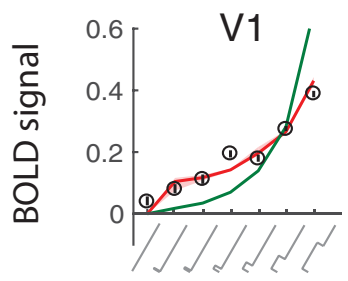

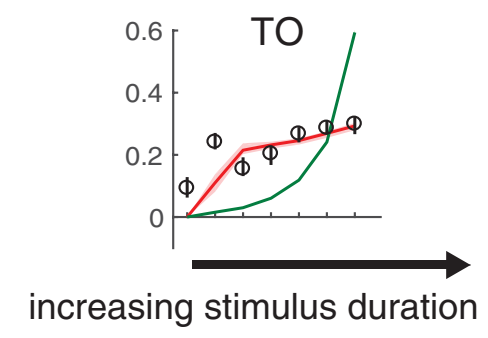

space
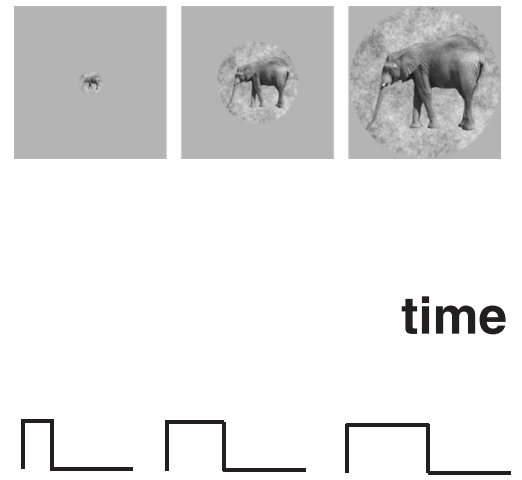

Figure 13. Subadditive spatial and temporal summation. $A, B O L D$ responses pooled across voxels in $V 1$ (left) and in $\mathrm{TO}$ (right) are plotted as a function of stimulus size. Circles and error bars represent means and SEs across bootstrapped estimates. A compressive spatial summation model (red), fit to separate data, predicts the responses slightly more accurately than a linear model (green) in V1, and substantially more accurately in T0. Adapted with permission from Kay et al. (2013a, their Fig. 8). B, A similar pattern is observed for duration, replotted from Figure 4 A.

dynamic response would become increasingly compressive along the visual hierarchy. Third, because even our longest stimuli were brief ( $\leq 528 \mathrm{~ms}$ ), thereby eliciting relatively small BOLD signals $(\sim 0.5 \%)$, it is unlikely that saturation of the BOLD signal for longer stimulus durations could explain the compressive response. For example, when similar stimuli are presented in a sequence of several images, the fMRI responses are several times larger (1\%-4\%) (Kay et al., 2013a, b), indicating that the BOLD signal measured here was well below saturation. Therefore, overall, our results indicate that the neuronal response underlying the BOLD signal shows significant temporal subadditivities, and that the subadditivity is more pronounced in later visual areas.

Multiple studies are consistent with the possibility that the linear approximation of the neural-to-BOLD transform is reasonably good (Boynton et al., 1989; Heeger et al., 2000; Rees et al., 2000). However, our interpretation of temporal compressive summation in the neural response does not rely on the assumption that the BOLD signal is exactly a linear transform of local neuronal activity. If, for example, the coupling reflects an approximately square root compression, as recently suggested by one group (Bao et al., 2015), then the stimulus-to-BOLD nonlinearity we observed would still imply a highly compressive neural response. This is easiest to appreciate for the power-law implementation of the CTS model. For example, the median exponent fit to the BOLD signal across ROIs ranged from 0.1 (IPS) to 0.28 (V1). If we assume that this includes a neurovascular compressive exponent of 0.5 , then the stimulus-to-neural response would have exponents ranging from 0.2 (IPS) to 0.56 (V1), still highly compressive. This interpretation is supported by preliminary analyses

\section{$\leftarrow$}

(Figure legend continued.) and followed by scaling and summation to predict the BOLD signal, as indicated in Figure 3. Each of the three implementations is fit to the same data (Experiment 1, same as Fig. 4). $\boldsymbol{B}$, The summary metrics, $T_{\text {isi }}$ and $R_{\text {double, }}$ are similar for the three implementations, with a general tendency for V1-V3 (circled) to have shorter $\mathrm{T}_{\text {isi }}$ and higher $\mathrm{R}_{\text {double, }}$ indicated by the lower right position in the scatter plots. This shows that the three CTS implementations, despite different parameterizations, manifest in similar model behavior. C, Cross-validated accuracy is high for all three model forms, well above the linear model for all areas. Figure made from the script trf_mkFigure12.m. of intracranial data, which show substantial temporal nonlinearities in the neural response (Zhou et al., 2017).

\section{Spatial and temporal subadditivities}

Subadditive temporal summation is likely to have important functional consequences. The two ways we documented temporal subadditivities, a compressive function of duration for single stimuli and a reduced response for paired stimuli with short ISIs, are consistent with neural adaptation: a reduced response to prolonged or repeated stimuli. These phenomena are thought to reflect adaptive changes to the local environment, rather than being a passive byproduct of neural responses (Webster, 2015). For example, adaptation may serve to prioritize new information or act as gain control (Solomon and Kohn, 2014). An interesting consequence of subadditive temporal summation is that responses to stimuli of different durations are more similar to one another than they would be if summation were linear. This may be thought of as a form of duration or timing tolerance, analogous to size and position tolerance in spatial encoding, which are increasingly prominent in higher visual areas (Kay et al., 2013a). For example, in V1, as the stimulus size increases or the stimulus duration lengthens, the response amplitude increases substantially, whereas in area TO, the response amplitudes increase only slightly, indicating greater tolerance for size and duration (Fig. 13).

Although spatial and temporal subadditivities share some properties, they are independent findings and differ in detail. For example, V2 shows substantially more spatial subadditivity than V1 (Kay et al., 2013b, their Fig. 9b; Kay et al., 2013a, their Fig. 7b), but a similar degree of temporal subadditivity (Figs. 6, 7). Moreover, temporal subadditivities are directional: the future cannot affect the past, whereas responses to two spatial locations can affect each other. Further, a system that is space-time separable could, in principle, exhibit saturation with space but be linear in time, or vice versa. It will be important in future work to develop an integrated model that accounts for spatial and temporal nonlinearities.

\section{Temporal window length}

Our finding that time scales lengthen across the visual hierarchy is consistent with measurements of temporal dynamics at a larger 
scale. For example, temporal receptive window length was studied by measuring response reliability to scrambled movie segments (Hasson et al., 2008; Honey et al., 2012). In visual cortex, responses depended on information accumulated over $\sim 1 \mathrm{~s}$, whereas in anterior temporal, parietal, and frontal areas the time scale ranged from $\sim 12$ to $36 \mathrm{~s}$. Similarly, in event-related fMRI, the influence of prior trials was modeled with an exponential decay, with longer time constants in later areas: Boynton et al. (1996) reported a time constant of $\sim 1 \mathrm{~s}$ in V1 for contrast reversing checkerboards, and Mattar et al. (2016), using static face images, reported short time constants in V1 $(\sim 0.6 \mathrm{~s})$ and much longer constants in face areas $(\sim 5 \mathrm{~s})$. In macaque, the timescale of autocorrelations in spike counts was longer for areas higher in the hierarchy $(\sim 300 \mathrm{~ms})$ compared with sensory areas $(\sim 75-100$ ms) (Murray et al., 2014). These studies used very different methods and resulted in a wide range of time-scale estimates. It will be important in future work to ask whether a forward model can account for the range of values.

Analyzing visual information at multiple temporal scales has benefits. First, accumulating information in the past is necessary for predicting the future, and a hierarchy of temporal windows may be useful for predictions over different time-scales (Heeger, 2017). Second, signal-to-noise ratios are optimized when the temporal scale of analysis is matched to the temporal scale of the event of interest (a "matched filter"); different visual areas extract information about different image properties, which in turn are likely to have different temporal (or spatiotemporal) distributions in natural viewing. For example, V1 cells are highly sensitive to the spatially local orientation, contrast, and spatial frequency in an image. These properties are likely to change with even small eye movements, such that integrating over too long a time period will blur the dimensions of interest. In contrast, higher-order image statistics may be stable over larger image regions and longer viewing durations, and hence an area sensitive to such properties may benefit from longer periods of integration. Whether or not the time scales of the different cortical areas are fixed, or adjust based on the ongoing statistics of visual input, is an important question for future work.

Just as understanding natural image statistics may lead to better theories of neural coding (Olshausen and Field, 1996; Schwartz and Simoncelli, 2001), understanding neural coding can help us understand behavior. For example, the time-scale of cortical areas may set the time-scale of integration for behavior. Words, faces, and global motion patterns are integrated over periods 5-10 times longer than textures and local motion patterns (Holcombe, 2009). These effects have not been connected to a neural model; modeling the time-scale of cortical areas critical for these tasks may help explain these large behavioral effects.

\section{Generalization and future directions}

The CTS model parameters estimated from our main experiment are similar to those from the second experiment (self-replication), in which we used different stimulus images. Yet, just as with spatial pRF models, it is likely that our model will fail for certain tasks or stimuli (Wandell and Winawer, 2015). For example, sustained attention to the stimulus (Self et al., 2016), presence of a surround (Bair et al., 2003), nonseparable spatiotemporal patterns (motion), and stimulus history of many seconds or more (Weiner et al., 2010), can all affect the time course, hence subadditivity of the response. By formulating a forward model of responses to large-field contrast stimuli during passive viewing, we provide a quantitative benchmark that can be used to guide interpretation of how other factors influence response dynamics, and a platform upon which to extend the model to new stimulus or task features. An important goal for future work is to develop a space-time model that simultaneously accounts for nonlinearities in spatial (Kay et al., 2013a) and temporal summation. Finally, our fMRI model contains a static nonlinearity. Measurements with finer temporal resolution, such as intracranial EEG, will be informative for understanding the time scale of the nonlinearities.

\section{References}

Adelson EH, Bergen JR (1985) Spatiotemporal energy models for the perception of motion. J Opt Soc Am A 2:284-299. CrossRef Medline

Bair W, Cavanaugh JR, Movshon JA (2003) Time course and time-distance relationships for surround suppression in macaque V1 neurons. J Neurosci 23:7690-7701. Medline

Bao P, Purington CJ, Tjan BS (2015) Using an achiasmic human visual system to quantify the relationship between the fMRI BOLD signal and neural response. Elife 4:e09600. CrossRef Medline

Boynton GM, Engel SA, Glover GH, Heeger DJ (1996) Linear systems analysis of functional magnetic resonance imaging in human V1. J Neurosci 16:4207-4221. Medline

Boynton GM, Engel SA, Heeger DJ (2012) Linear systems analysis of the fMRI signal. Neuroimage 62:975-984. CrossRef Medline

Boynton RM, Fargo L, Olson CX, Smallman HS (1989) Category effects in color memory. Color Res Application 14:229-234. CrossRef

Brainard DH (1997) The Psychophysics Toolbox. Spat Vis 10:433-436. CrossRef Medline

Britten KH, Heuer HW (1999) Spatial summation in the receptive fields of MT neurons. J Neurosci 19:5074-5084. Medline

Carandini M, Heeger DJ (2011) Normalization as a canonical neural computation. Nat Rev Neurosci 13:51-62. CrossRef Medline

DiCarlo JJ, Zoccolan D, Rust NC (2012) How does the brain solve visual object recognition? Neuron 73:415-434. CrossRef Medline

Dong DW, Atick JJ (1995) Statistics of natural time-varying images. Netw Comp Neural 6:345-358. CrossRef

Dumoulin SO, Wandell BA (2008) Population receptive field estimates in human visual cortex. Neuroimage 39:647-660. CrossRef Medline

Friston KJ, Fletcher P, Josephs O, Holmes A, Rugg MD, Turner R (1998) Event-related fMRI: characterizing differential responses. Neuroimage 7:30-40. CrossRef Medline

Gauthier I, Skudlarski P, Gore JC, Anderson AW (2000) Expertise for cars and birds recruits brain areas involved in face recognition. Nat Neurosci 3:191-197. CrossRef Medline

Grill-Spector K, Kourtzi Z, Kanwisher N (2001) The lateral occipital complex and its role in object recognition. Vision Res 41:1409-1422. CrossRef Medline

Hasson U, Yang E, Vallines I, Heeger DJ, Rubin N (2008) A hierarchy of temporal receptive windows in human cortex. J Neurosci 28:2539-2550. CrossRef Medline

Heckman GM, Bouvier SE, Carr VA, Harley EM, Cardinal KS, Engel SA (2007) Nonlinearities in rapid event-related fMRI explained by stimulus scaling. Neuroimage 34:651-660. CrossRef Medline

Heeger DJ (1992) Normalization of cell responses in cat striate cortex. Vis Neurosci 9:181-197. CrossRef Medline

Heeger DJ (2017) Theory of cortical function. Proc Natl Acad Sci U S A 114:1773-1782. CrossRef Medline

Heeger DJ, Simoncelli EP, Movshon JA (1996) Computational models of cortical visual processing. Proc Natl Acad Sci U S A 93:623-627. CrossRef Medline

Heeger DJ, Huk AC, Geisler WS, Albrecht DG (2000) Spikes versus BOLD: what does neuroimaging tell us about neuronal activity? Nat Neurosci 3:631-633. CrossRef Medline

Helmholtz H (1886) Helmholtz's treatise on physiological optics. Rochester, NY: Optical Society of America.

Hess RF, Plant GT (1985) Temporal frequency discrimination in human vision: evidence for an additional mechanism in the low spatial and high temporal frequency region. Vision Res 25:1493-1500. CrossRef Medline

Heuer HW, Britten KH (2002) Contrast dependence of response normalization in area MT of the rhesus macaque. J Neurophysiol 88:3398-3408. CrossRef Medline

Holcombe AO (2009) Seeing slow and seeing fast: two limits on perception. Trends Cogn Sci 13:216-221. CrossRef Medline 
Honey CJ, Thesen T, Donner TH, Silbert LJ, Carlson CE, Devinsky O, Doyle WK, Rubin N, Heeger DJ, Hasson U (2012) Slow cortical dynamics and the accumulation of information over long timescales. Neuron 76:423434. CrossRef Medline

Horiguchi H, Nakadomari S, Misaki M, Wandell BA (2009) Two temporal channels in human V1 identified using fMRI. Neuroimage 47:273-280. CrossRef Medline

Kastner S, De Weerd P, Pinsk MA, Elizondo MI, Desimone R, Ungerleider LG (2001) Modulation of sensory suppression: implications for receptive field sizes in the human visual cortex. J Neurophysiol 86:1398-1411. Medline

Kay KN (2017) Principles for models of neural information processing. Neuroimage. Advance online publication. Retrieved Aug. 6, 2017. CrossRef Medline

Kay KN, Winawer J, Mezer A, Wandell BA (2013a) Compressive spatial summation in human visual cortex. J Neurophysiol 110:481-494. CrossRef Medline

Kay KN, Winawer J, Rokem A, Mezer A, Wandell BA (2013b) A two-stage cascade model of BOLD responses in human visual cortex. PLoS Comput Biol 9:e1003079. CrossRef Medline

Kay KN, Rokem A, Winawer J, Dougherty RF, Wandell BA (2013c) GLMdenoise: a fast, automated technique for denoising task-based fMRI data. Front Neurosci 7:247. CrossRef Medline

Kay KN, Weiner KS, Grill-Spector K (2015) Attention reduces spatial uncertainty in human ventral temporal cortex. Curr Biol 25:595-600. CrossRef Medline

Koenderink JJ, van de Grind WA, Bouman MA (1972) Opponent color coding: a mechanistic model and a new metric for color space. Kybernetik 10:78-98. CrossRef Medline

Kohn A (2007) Visual adaptation: physiology, mechanisms, and functional benefits. J Neurophysiol 97:3155-3164. CrossRef Medline

Marr D (1982) Vision: a computational investigation into the human representation and processing of visual information. San Francisco: Freeman.

Mattar MG, Kahn DA, Thompson-Schill SL, Aguirre GK (2016) Varying timescales of stimulus integration unite neural adaptation and prototype formation. Curr Biol 26:1669-1676. CrossRef Medline

Maunsell JH, Newsome WT (1987) Visual processing in monkey extrastriate cortex. Annu Rev Neurosci 10:363-401. CrossRef Medline

McKee SP, Taylor DG (1984) Discrimination of time: comparison of foveal and peripheral sensitivity. J Opt Soc Am A 1:620-627. CrossRef Medline

Motter BC (2006) Modulation of transient and sustained response components of $\mathrm{V} 4$ neurons by temporal crowding in flashed stimulus sequences. J Neurosci 26:9683-9694. CrossRef Medline

Murray JD, Bernacchia A, Freedman DJ, Romo R, Wallis JD, Cai X, PadoaSchioppa C, Pasternak T, Seo H, Lee D, Wang XJ (2014) A hierarchy of intrinsic timescales across primate cortex. Nat Neurosci 17:1661-1663. CrossRef Medline

Olshausen BA, Field DJ (1996) Emergence of simple-cell receptive field properties by learning a sparse code for natural images. Nature 381:607609. CrossRef Medline

Pelli DG (1997) The VideoToolbox software for visual psychophysics: transforming numbers into movies. Spat Vis 10:437-442. CrossRef Medline

Priebe NJ, Churchland MM, Lisberger SG (2002) Constraints on the source of short-term motion adaptation in macaque area MT: I. The role of input and intrinsic mechanisms. J Neurophysiol 88:354-369. CrossRef Medline

Rees G, Friston K, Koch C (2000) A direct quantitative relationship between the functional properties of human and macaque V5. Nat Neurosci 3:716-723. CrossRef Medline

Rodieck RW, Binmoeller KF, Dineen J (1985) Parasol and midget ganglion cells of the human retina. J Comp Neurol 233:115-132. CrossRef Medline

Rolls ET, Tovee MJ (1995) The responses of single neurons in the temporal visual cortical areas of the macaque when more than one stimulus is present in the receptive field. Exp Brain Res 103:409-420. Medline

Rust NC, Dicarlo JJ (2010) Selectivity and tolerance ("invariance") both increase as visual information propagates from cortical area V4 to IT. J Neurosci 30:12978-12995. CrossRef Medline

Rust NC, DiCarlo JJ (2012) Balanced increases in selectivity and tolerance produce constant sparseness along the ventral visual stream. J Neurosci 32:10170-10182. CrossRef Medline

Schwartz O, Simoncelli EP (2001) Natural signal statistics and sensory gain control. Nat Neurosci 4:819-825. CrossRef Medline

Self MW, Peters JC, Possel JK, Reithler J, Goebel R, Ris P, Jeurissen D, Reddy L, Claus S, Baayen JC, Roelfsema PR (2016) The effects of context and attention on spiking activity in human early visual cortex. PLoS Biol 14:e1002420. CrossRef Medline

Simoncelli EP, Heeger DJ (1998) A model of neuronal responses in visual area MT. Vision Res 38:743-761. CrossRef Medline

Snow M, Coen-Cagli R, Schwartz O (2016) Specificity and timescales of cortical adaptation as inferences about natural movie statistics. J Vis 16: 13. CrossRef Medline

Solomon SG, Kohn A (2014) Moving sensory adaptation beyond suppressive effects in single neurons. Curr Biol 24:R1012-R1022. CrossRef Medline

Stigliani A, Jeska B, Grill-Spector K (2017) An encoding model of temporal processing in human visual cortex. bioRxiv 108985. CrossRef

Tolhurst DJ, Walker NS, Thompson ID, Dean AF (1980) Non-linearities of temporal summation in neurones in area 17 of the cat. Exp Brain Res 38:431-435. Medline

Tovee MJ, Rolls ET, Azzopardi P (1994) Translation invariance in the responses to faces of single neurons in the temporal visual cortical areas of the alert macaque. J Neurophysiol 72:1049-1060. Medline

Wandell BA (1995) Foundations of vision. Sunderland, MA: Sinauer.

Wandell BA, Winawer J (2015) Computational neuroimaging and population receptive fields. Trends Cogn Sci 19:349-357. CrossRef Medline

Wang L, Mruczek RE, Arcaro MJ, Kastner S (2015) Probabilistic maps of visual topography in human cortex. Cereb Cortex 25:3911-3931. CrossRef Medline

Watson AB (1982) Derivation of the impulse response: comments on the method of Roufs and Blommaert. Vision Res 22:1335-1337. CrossRef Medline

Watson AB (1986) Temporal sensitivity. In: Handbook of perception and human performance (Boff KR, Kaufman L, Thomas JP, eds), pp 6.1-6.43. New York: Wiley.

Watson AB, Robson JG (1981) Discrimination at threshold: labelled detectors in human vision. Vision Res 21:1115-1122. CrossRef Medline

Webster MA (2015) Visual adaptation. Annu Rev Vis Sci 1:547-567. CrossRef Medline

Weiner KS, Grill-Spector K (2010) Sparsely-distributed organization of face and limb activations in human ventral temporal cortex. Neuroimage 52: 1559-1573. CrossRef Medline

Weiner KS, Sayres R, Vinberg J, Grill-Spector K (2010) fMRI-adaptation and category selectivity in human ventral temporal cortex: regional differences across time scales. J Neurophysiol 103:3349-3365. CrossRef Medline

Weiss Y, Simoncelli EP, Adelson EH (2002) Motion illusions as optimal percepts. Nat Neurosci 5:598-604. CrossRef Medline

Winawer J, Parvizi J (2016) Linking electrical stimulation of human primary visual cortex, size of affected cortical area, neuronal responses, and subjective experience. Neuron 92:1213-1219. CrossRef Medline

Winawer J, Kay KN, Foster BL, Rauschecker AM, Parvizi J, Wandell BA (2013) Asynchronous broadband signals are the principal source of the BOLD response in human visual cortex. Curr Biol 23:1145-1153. CrossRef Medline

Worsley KJ, Liao CH, Aston J, Petre V, Duncan GH, Morales F, Evans AC (2002) A general statistical analysis for fMRI data. Neuroimage 15:1-15. CrossRef Medline

Zhou J, Benson NC, Kay K, Winawer J (2017) Modeling systematic differences in temporal summation and adaptation in human visual cortex: evidence from fMRI and intracranial EEG. bioRxiv. CrossRef 